\title{
Bisphenol $A$ and the risk of cardiometabolic disorders: a systematic review with meta- analysis of the epidemiological evidence
}

Fanny Rancière ${ }^{1,2,3 \dagger}$, Jasmine G. Lyons ${ }^{3+}$, Venurs H. Y. Loh ${ }^{3}$, Jérémie Botton ${ }^{1,2,4}$, Tamara Galloway ${ }^{5}$, Tiange Wang ${ }^{6}$, Jonathan E. Shaw ${ }^{3}$ and Dianna J. Magliano ${ }^{3 *}$

\begin{abstract}
Bisphenol A (BPA) is suspected to be associated with several chronic metabolic diseases. The aim of the present study was to review the epidemiological literature on the relation between BPA exposure and the risk of cardiometabolic disorders. PubMed and Embase databases were searched up to August 2014 by two independent investigators using standardized subject terms. We included observational studies (cohort, case-control and cross-sectional studies) carried out in children or adults, measuring urinary BPA (UBPA), including at least 100 participants and published in English. The health outcomes of interest were diabetes, hyperglycemia, measures of anthropometry, cardiovascular disease (CVD) and hypertension. Data were extracted and meta-analyzed when feasible, using a random-effects model. Thirty-three studies with sample size ranging from 239 to 4811 met the inclusion criteria, including five with a prospective design. Twelve studies reported on diabetes or hyperglycemia, 16 on anthropometry, 6 on CVD and 3 on hypertension. Evidence for a positive association between uBPA concentrations and diabetes, overweight, obesity, elevated waist circumference (WC), CVD and hypertension was found in $7 / 8,2 / 7,6 / 7,5 / 5,4 / 5$ and 2/3 of the cross-sectional studies, respectively. We were able to conduct outcome-specific meta-analyses including 12 studies. When comparing the highest vs. the lowest uBPA concentrations, the pooled ORs were 1.47 (95 \% Cl: 1.21-1.80) for diabetes, 1.21 (95\% Cl: 0.98-1.50) for overweight, 1.67 (95\% Cl: 1.41-1.98) for obesity, 1.48 (95\% Cl: 1.25-1.76) for elevated WC, and 1.41 (95\% Cl: 1.12-1.79) for hypertension. Moreover, among the five prospective studies, 3 reported significant findings, relating BPA exposure to incident diabetes, incident coronary artery disease, and weight gain. To conclude, there is evidence from the large body of cross-sectional studies that individuals with higher uBPA concentrations are more likely to suffer from diabetes, general/abdominal obesity and hypertension than those with lower uBPA concentrations. Given the potential importance for public health, prospective cohort studies with proper adjustment for dietary characteristics and identification of critical windows of exposure are urgently needed to further improve knowledge about potential causal links between BPA exposure and the development of chronic disease.
\end{abstract}

Keywords: Bisphenol A, Cardiovascular disease, Diabetes, Epidemiology, Hyperglycemia, Hypertension, Meta-analysis, Obesity, Overweight, Systematic review

\footnotetext{
* Correspondence: dianna.magliano@bakeridi.edu.au

${ }^{\dagger}$ Equal contributors

${ }^{3}$ Department of Clinical Diabetes and Epidemiology, Baker IDI Heart and Diabetes Institute, Level 4, 99 Commercial Road, Melbourne, VIC 3000,

Australia

Full list of author information is available at the end of the article
}

\section{Biomed Central}

(c) 2015 Rancière et al. This is an Open Access article distributed under the terms of the Creative Commons Attribution License (http://creativecommons.org/licenses/by/4.0), which permits unrestricted use, distribution, and reproduction in any medium, provided the original work is properly credited. The Creative Commons Public Domain Dedication waiver (http:// creativecommons.org/publicdomain/zero/1.0/) applies to the data made available in this article, unless otherwise stated. 


\section{Introduction}

In recent decades, large and rapid increases in diabetes and obesity prevalence have been observed worldwide. Apart from traditional risk factors such as family history, sedentary lifestyle and energy dense dietary intake, attention has recently turned to environmental toxicants called endocrine-disrupting chemicals (EDCs) because of their ability to interfere with synthesis, secretion, transport, metabolism, binding action, or elimination of natural blood-borne hormones, and to induce obesogenic or diabetogenic effects [1]. In particular, bisphenol A (BPA) has been strongly suspected as a potential contributor to these disease aetiologies [2-5].

BPA is a synthetic monomer used in the manufacture of polycarbonate plastics and epoxy resins, with a world production estimated at 3.8 million tons in 2006 [6]. Importantly, this production of BPA is expected to increase further in the coming years, given the robust demand for polycarbonate plastics and epoxy resins from China [7] and other emerging markets. The primary source of human exposure to BPA is presumed to be via the ingestion of food which has been stored or reheated in BPA-lined containers, but recent data suggest there is at least some exposure from drinking water, dental sealants, thermal paper and, to a lesser extent, inhalation of household dust particles [8-12]. BPA is ubiquitous in our environment, as evidenced by the fact that over $90 \%$ of individuals have detectable levels of BPA present in their urine [13], which is the primary route of excretion in humans [14]. BPA has been found in neonates, children and adults [13], and can be measured in a range of bodily fluids and tissues, including urine, blood, saliva, placental tissue, adipose tissue and breast milk $[13,15]$.

There is accumulating in vitro and animal data (small and large animal) supporting a role of BPA in the development of diabetes, supporting a role of BPA in the development of diabetes, cardiovascular disease (CVD) and obesity. BPA is structurally similar to $17 \beta$-estradiol and thus binds to estrogen-related receptors (ER) such as ER $\alpha, E R \beta$ and ER $\gamma$, the G protein-coupled estrogen receptor GPR30, and the peroxisome proliferator-activated receptor gamma (PPAR- $\gamma$ ) [16, 17]. While the mechanisms of action are not fully understood, binding of BPA to these receptors has been shown to induce insulin resistance, adipogenesis, pancreatic beta-cell dysfunction, inflammation, and oxidative stress $[3,18-20]$. At concentrations typically seen in humans, BPA has been shown to act via extranuclear ER $\alpha$ [21] and ER $\beta$ [22]. These two studies used $\beta$-cells and whole islets of Langerhans from mice lacking ER $\alpha$ and ER $\beta$ and humans to demonstrate that the low dose effect of $\mathrm{BPA}$ is mediated via these estrogen receptors. Other experimental studies have also shown that BPA at environmentally relevant doses could inhibit the release of adiponectin, an adipokine that protects humans from obesity-related metabolic syndrome [23]. It also may have direct proangiogenic effects on human primary endothelial cells, suggesting that the human endothelium may be an important target for BPA [24]. Recently, Marmugi et al. showed that BPA exposure for 8 months in adult mice resulted in increased adipose tissue mass, hyperglycaemia, glucose intolerance, hypercholesterolemia and increased cholesterol biosynthesis by the liver [25]. However, the relevance of animal studies to humans remains unclear due to enterohepatic recirculation in rodents, resulting in a slower rate of BPA excretion compared with humans [14].

Given the ongoing policy debate on the possible public health benefits of minimizing BPA exposure, there is an urgent need for research to adequately evaluate cardiometabolic health in relation to BPA exposure. The past 10 years have seen a rapid increase in published reports of human, population-based epidemiological studies linking BPA to obesity, diabetes and CVD, most of which are cross-sectional studies. To date, three reviews have evaluated the available literature concerning BPA and chronic disease in humans [26-28]. However, the authors did not perform metaanalysis and, as the body of literature is growing rapidly, important new evidence can appear within short timelines [29]. Thus the aims of the present review were:

1. to provide insight into the most recent epidemiological evidence on the association between urinary BPA ( $\mathrm{uBPA}$ ) concentrations and chronic, cardiometabolic disorders (diabetes, overweight, obesity, CVD and hypertension);

2. to carry out meta-analysis when feasible; and

3. to identify the gaps in research that will allow for a comprehensive assessment of possible risk to humans.

\section{Methods}

This review follows PRISMA guidelines for systematic reviews of observational studies [30].

\section{Eligibility criteria}

Studies were included if the following inclusion criteria were met:

- Participants - Human studies including adults or children (however only diabetes in adults, in an attempt to limit the analysis to type 2)

- Outcomes - Studies examining diabetes, hyperglycemia, measures of anthropometry/ adiposity, CVD or hypertension. Surrogate cardiometabolic outcomes such as blood cholesterol levels and insulin sensitivity indices (e.g., Homeostasis Model Assessment of Insulin 
Resistance [HOMA], Quantitative Insulin Sensitivity Check Index [QUICKI], Matsuda Index) were not considered.

- Exposure - Urinary measures of BPA and/or BPA metabolites (studies examining BPA exposure in pregnant women in relation to outcome in the offspring were beyond the scope of this review)

- Sample size - Studies including at least 100 participants,

- Language - Studies published in English,

- Appropriate statistical adjustment - Models including adjustment for confounders (at least age and gender).

\section{Information sources and search}

PubMed and Embase databases were searched to identify potential studies for this review published up to August 2014. The keywords (and corresponding Medical Subject Heading $[\mathrm{MeSH}]$ terms for the search in PubMed) 'body mass index,' 'overweight, 'obesity', 'waist circumference,' 'body weight', 'abdominal obesity', 'cardiovascular disease', 'coronary heart disease,' 'diabetes,' 'hypertension, 'blood pressure, 'insulin resistance,' 'glucose intolerance' were combined with the Boolean operator 'OR'. The key term 'Bisphenol A' was entered and combined with the former using the Boolean operator 'AND'. The full electronic search strategy in PubMed is reported in the Additional file 1: Figure S1. References from identified studies were handsearched to ensure that no relevant studies were missed.

\section{Study selection and data extraction}

All the identified publications were evaluated for relevance by two independent reviewers (DJM, FR), on the basis of their titles and abstracts; any disagreement was resolved by discussion. Full texts of the selected abstracts were then checked for the inclusion criteria by the reviewers. Final eligibility of studies was decided by consensus.

Summary data for included studies were extracted into a standardized tool that included: study design, country, population, sample size, age, gender, ethnicity, outcome definition, method of exposure measurement, uBPA levels and categorization, results expressed as adjusted odds ratios (OR), hazard ratios (HR) or $\beta$-coefficients, and variables used in adjustment.

The quality of individual studies, with regard to the outcomes of interest, was independently assessed by two investigators. We utilised a scoring system based on the established OHAT guidelines [31] adapted to reflect the characteristics of the included studies: longitudinal design ( 2 points), population-based study (1 point), outcome assessment including measurements (1 point), collection of at least 2 urine samples per participant ( 1 point), control for urine dilution (1 point), adjustment for dietary intake (1 point), and adjustment for socioeconomic variables (1 point). Studies were then classified as 'low quality' (total score between 0 and 2), 'medium quality' (total score between 3 and 5), or 'high quality' (total score between 6 and 8 ).

\section{Meta-analysis}

We appraised each study to examine sources of heterogeneity, including difference in clinical outcomes and exposure measurements. Participant overlap between related studies was examined. For studies that used similar sources of data (e.g., surveys of National Health and Nutrition Examination Survey [NHANES], over concurrent periods and similar age range), only the study with the largest data set was included in the meta-analysis.

For each outcome, two studies with comparable outcome and exposure definitions were sufficient to perform a meta-analysis. For anthropometric outcomes, we performed pooled and separate analysis for children and adults. ORs with corresponding confidence intervals (CIs) were extracted from the most adjusted model. For summary purposes, we pooled OR estimates comparing extreme categories of uBPA levels (the highest vs. lowest uBPA levels). A random effects model was used. The logarithm of the OR and its standard error (SE) was calculated using the formula of log (upper limit of $95 \% \mathrm{CI}$ ) minus $\log$ (lower limit of $95 \% \mathrm{CI}$ ) divided by 3.92 (2*1.96, the 97.5 th percentile of the standard normal distribution) and was entered into REVMAN 5.1 software (REVMAN 2011). Heterogeneity between studies was tested using Chi-squared test and quantified by calculating the $\mathrm{I}^{2}$ statistic. We produced forest plots to visually assess the individual study ORs and overall ORs, with corresponding $95 \%$ CIs.

\section{Results \\ Study selection}

The initial search identified 953 studies in total. Of these, 895 studies were excluded after screening of the titles/abstracts (studies did not meet inclusion criteria e.g., were animal or in vitro studies, reported only serum BPA levels, or were review/commentary articles). Fifty eight studies were thus identified and reviewed in fulltext versions, from which 33 studies were selected to be included in the systematic review (Fig. 1).

Twelve independent cross-sectional studies were selected for quantitative synthesis, making meta-analysis possible for 5 health outcomes: diabetes, overweight, obesity, elevated waist circumference (WC) and hypertension. Individual and pooled OR estimates are shown in the Additional file 1: Figure S2. Studies included and excluded from meta-analysis and reasons for exclusion are summarized in the Additional file 1: Table S1. 


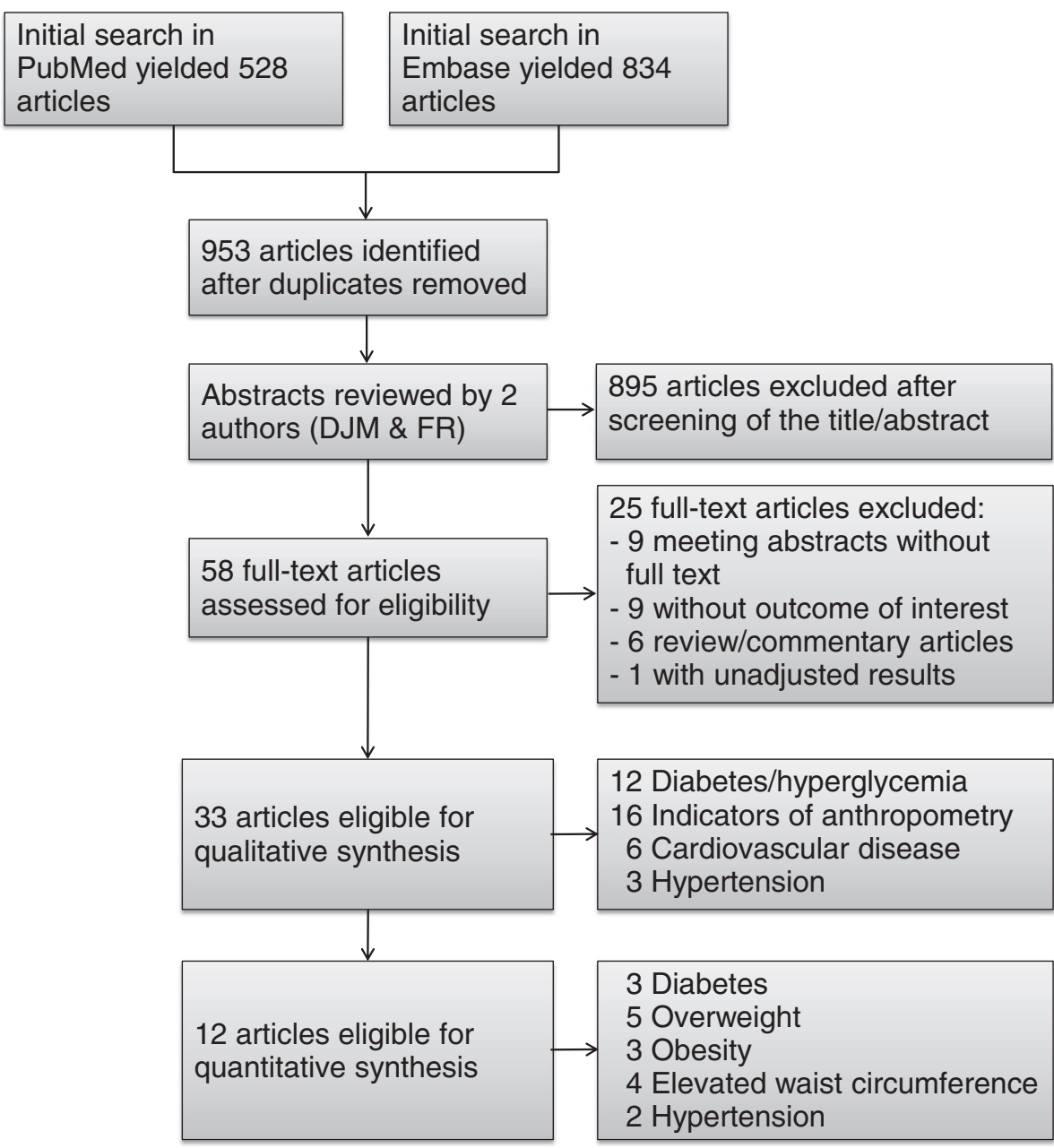

Fig. 1 Flow diagram of the search strategy for the systematic review (some studies reported on several outcomes)

\section{Characteristics of studies}

Characteristics for each of the 33 articles included in the review are summarized in Table 1 and detailed in the Additional file 1: Table S2.

\section{Study design and population}

This systematic review includes 28 cross-sectional and 5 longitudinal studies, drawn from 19 different study populations and 6 different countries. Sample sizes ranged from 239 [32] to 4811 [33] representing a total of 69,486 participants, not accounting for participant overlap across publications utilizing data from the same study populations.

Among the cross-sectional studies, 16 (57 \%) used study populations from the NHANES study (cycles ranging from the years 2003 to 2010) [33-48]. Potential overlap between all publications using data from NHANES is summarized in the Additional file 1: Table S3. Five cross-sectional studies (18\%) used Chinese populations [49-53], including two studies using the same population sample from Songnan Community in Shanghai $[51,52]$. Four cross-sectional studies $(14 \%)$ used a Korean population [54-57], while other single studies were conducted in Italians [58], Iranians [32], and British populations [59]. Of the 5 prospective studies, one was on the EPIC-Norfolk study (UK) [60] and 4 from U.S. cohorts, NHS and NHSII [61, 62], HOME [63] and CHAMACOS [64].

Of the 16 NHANES publications, 11 investigated adults [34, 36, 37, 39-46], while four restricted their study population to children/teenagers [35, 38, 47, 48]. One further study examined adults for CVD outcomes; however this study was excluded from the diabetes outcome analysis because the study population included a mixed sample of children and adults [33]. Of the 12 other cross-sectional studies, 10 were conducted in adult populations [32, 51-59], and 2 in school-age children $[49,50]$. Three of the prospective studies were conducted in adults [60-62]. Of the two in children $[63,64]$, follow-up for the infants in the HOME study 
Table 1 Summary characteristics of studies included in the systematic review ( $n=33$ studies)

\begin{tabular}{|c|c|}
\hline Study characteristics & $n$ \\
\hline \multicolumn{2}{|l|}{ Year of publication } \\
\hline 2008 & 1 \\
\hline 2009 & 0 \\
\hline 2010 & 2 \\
\hline 2011 & 5 \\
\hline 2012 & 10 \\
\hline 2013 & 8 \\
\hline 2014 & 7 \\
\hline \multicolumn{2}{|l|}{$\begin{array}{l}\text { Geographical } \\
\text { setting }\end{array}$} \\
\hline Asia & 10 \\
\hline Europe & 3 \\
\hline North America & 20 \\
\hline \multicolumn{2}{|l|}{ Design } \\
\hline Cross-sectional & 28 \\
\hline Prospective & 5 \\
\hline \multicolumn{2}{|l|}{ Age category } \\
\hline Children & 8 \\
\hline Adults & 25 \\
\hline \multicolumn{2}{|l|}{ Gender } \\
\hline Mixed & 30 \\
\hline Women only & 3 \\
\hline \multicolumn{2}{|l|}{ Sample size } \\
\hline$<500$ & 5 \\
\hline $500-1000$ & 4 \\
\hline $1001-2000$ & 9 \\
\hline $2001-3000$ & 6 \\
\hline $3001-4000$ & 6 \\
\hline $4001-5000$ & 3 \\
\hline \multicolumn{2}{|l|}{ Health outcomes ${ }^{a}$} \\
\hline Diabetes & 9 \\
\hline Prediabetes & 1 \\
\hline Hyperglycemia & 2 \\
\hline Overweight & 7 \\
\hline Obesity & 7 \\
\hline $\begin{array}{l}\text { Elevated waist } \\
\text { circumference }\end{array}$ & 5 \\
\hline $\begin{array}{l}\text { Cardiovascular } \\
\text { disease }\end{array}$ & 6 \\
\hline Hypertension & 3 \\
\hline \multicolumn{2}{|l|}{ Urinary sample } \\
\hline Spot sample & 27 \\
\hline $\begin{array}{l}\text { First-morning-void } \\
\text { sample }\end{array}$ & 3 \\
\hline
\end{tabular}
25

Table 1 Summary characteristics of studies included in the systematic review ( $n=33$ studies) (Continued)

\begin{tabular}{ll} 
Second-morning & 1 \\
void sample & \\
12-h sample & 1 \\
$24-h$ sample & 1 \\
\hline Some studies reported on several outcomes
\end{tabular}

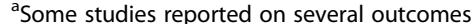

was at age 1 and 2 years [63], and for children from the CHAMACOS cohort at 5 and 9 years old [64].

All NHANES cycles as well as the NHS, NHSII and HOME cohorts comprised multiple ethnicities, but were predominantly non-Hispanic white. Mothers of children from the CHAMACOS cohort were mostly Latina. In 10 publications, all participants were Asian, from China [49-53], South Korea [54-57] or Iran [32].

\section{Outcomes}

This review included 12 publications on abnormal glucose tolerance $(9,2$ and 1 with outcomes of diabetes, hyperglycemia and prediabetes, respectively), 16 publications on anthropometry (7 with an outcome of overweight, 7 with obesity and 5 with elevated WC), 6 publications on CVD and 3 on hypertension.

\section{Urinary BPA assessment and levels}

All the studies used total (unconjugated and conjugated) uBPA concentration as the exposure variable. The majority of studies relied on a single uBPA measurement, however 3 assessed BPA exposure using repeated measurements: 2 follow-up assessments in paediatric studies $[63,64]$, and up to 5 measurements during the study period in an adult study [54].

The highest uBPA mean concentration was reported in a study in children within the NHANES 2003/08 cycles [35], with a mean (SE) of $5.0(0.3) \mathrm{ng} / \mathrm{mL}$ in boys and $4.6(0.3) \mathrm{ng} / \mathrm{mL}$ in girls. The earliest NHANES (2003/2004) study had the highest mean levels of BPA, and median BPA concentration followed a downward trend across subsequent NHANES cycles [37]. The lowest uBPA concentration was reported in a crosssectional study conducted in Chinese school children in 2011 [50], with a median (interquartile range, IQR) of $0.60(0.20-1.37) \mathrm{ng} / \mathrm{mL}$.

\section{Covariates}

Tables 2, 3 and 4 describe all variables used for the adjustment in the statistical analyses for each separate publication. All models were adjusted for at least age and gender. In order to correct for urine dilution, the majority of studies adjusted for an indicator of renal function, either urinary creatinine [33-40, 42, 45-48, 52, 55-57, 60-62, 64], estimated glomerular filtration rate (eGFR) [51], specific gravity [50], or serum creatinine [32]. Four studies 
Table 2 Summary of results in studies used as primary data: diabetes, prediabetes and hyperglycemia ( $n=12$ publications)

\begin{tabular}{|c|c|c|c|}
\hline Reference & Outcomes \& definitions used & Urinary BPA categorisation & Main results \\
\hline \multicolumn{4}{|c|}{ Prevalent diabetes (8 publications) } \\
\hline $\begin{array}{l}\text { Ahmadkhaniha et al. } 2014 \\
\text { [32] }\end{array}$ & $\begin{array}{l}\text { Type } 2 \text { diabetes: self-reported } \\
\text { and doctor-diagnosed T2D } \\
\text { according to the ADA guideline } \\
\text { (FPG }>126 \mathrm{mg} / \mathrm{dL} \text {, HbA1c }>6.5 \% \text { ) } \\
\text { for more than one year }\end{array}$ & $\begin{array}{l}\text { BPA in two groups based on the median } \\
(<0.85 \text { and } \geq 0.85 \mu \mathrm{g} / \mathrm{L})\end{array}$ & $\begin{array}{l}\mathrm{OR}=57.6 \\
(21.1-157.05)\end{array}$ \\
\hline \multirow[t]{14}{*}{ Casey \& Neidell 2013 [37] } & \multirow{14}{*}{$\begin{array}{l}\text { Diabetes: self-report of doctor } \\
\text { diagnosis }\end{array}$} & \multirow[t]{5}{*}{ BPA continuous (not log-transformed) } & Per SD increase: \\
\hline & & & 2003/04: OR = $1.398(1.183-1.653)$ \\
\hline & & & 2005/06: OR = $1.008(0.861-1.181)$ \\
\hline & & & $2007 / 08: \mathrm{OR}=0.716(0.500-1.025)$ \\
\hline & & & $\begin{array}{l}\text { Pooled 2003/08: OR = } 1.065(0.973- \\
1.166)\end{array}$ \\
\hline & & \multirow[t]{5}{*}{ BPA continuous (log-transformed) } & Per 10-fold increase: \\
\hline & & & 2003/04: OR = $1.492(1.267-1.757)$ \\
\hline & & & 2005/06: OR = $1.230(0.894-1.694)$ \\
\hline & & & 2007/08: OR = $0.932(0.759-1.146)$ \\
\hline & & & $\begin{array}{l}\text { Pooled 2003/08: OR = } 1.202 \text { (1.049- } \\
1.377)\end{array}$ \\
\hline & & \multirow{4}{*}{$\begin{array}{l}\text { BPA in quartiles (ng/mL): Q1: <1.2; Q2: } \\
\text { 1.2-2.2; Q3: 2.3-4.2; Q4: >4.2 }\end{array}$} & Pooled 2003/08: \\
\hline & & & Q2 vs. Q1: OR = $1.443(0.982-2.119)$ \\
\hline & & & Q3 vs. Q1: OR = $1.512(0.998-2.289)$ \\
\hline & & & Q4 vs. Q1: OR = $1.760(1.137-2.724)$ \\
\hline
\end{tabular}

Kim \& Park 2013 [56] doctor-diagnosed

\section{Diabetes: self-report of doctor} diagnosis

Melzer et al. $2010[40]$

Diabetes: self-report of doctor diagnosis

Type 2 diabetes: $F P G \geq 7.0 \mathrm{mmol} / \mathrm{L} \quad$ BPA in quartiles $(\mathrm{ng} / \mathrm{mL})$ : Q1: $\leq 0.47$; or plasma glucose $\geq 11.1 \mathrm{mmol} / \mathrm{L}$ two hours after oral glucose

Ning et al. 2011 [51]

Adjustment in model(s) used for review

Age, sex, BMl, hypertension, serum triglyceride level, serum cholesterol level, serum creatinine (smoking and consumption of sugared drinks in plastic bottles or canned food in two past weeks were exclusion criteria)

Age, sex, urinary creatinine concentration, race/ ethnicity, income, smoking, body mass index waist circumference, veteran/military status, citizenship status, marital status, household size, pregnancy status, language at subject interview, health insurance coverage, employment status in the prior week, consumption of bottled water in the past $24 \mathrm{~h}$, consumption of alcohol, annual consumption of tuna fish, presence of emotional support in one's life, being on a diet using a water treatment device, access to a routine source of health care, vaccinated for routine source of health care, vaccinated Hepatitis A or B, consumption of dietary
supplements (vitamins or minerals), inability to purchase balanced meals on a consistent basis + survey cycle for pooled analyses

Q2 vs. Q1: $O R=1.23(0.62-2.43)$

$\mathrm{Q} 3$ vs. $\mathrm{Q} 1: \mathrm{OR}=1.17(0.60-2.28)$

Q4 vs. Q1: $\mathrm{OR}=1.71(0.89-3.26)$

$p$ for trend $=0.374$

Per SD increase:

$\mathrm{OR}=1.39(1.21-1.60)$

Per SD increase:

2003/04: $\mathrm{OR}=1.40(1.25-1.56)$ 2005/06: $\mathrm{OR}=1.02(0.76-1.38)$

Pooled 2003/06: OR = $1.24(1.10$ 1.40) smoking, income, place of residence

Age, sex, race/ethnicity, education, income $\mathrm{BMI}, \mathrm{WC}$, smoking status, urinary creatinine

Age, gender, ethnicity, education, income, BMI, WC, smoking status, urinary creatinine

Age, sex, educational level, family history of diabetes, WC, systolic blood pressure, In(TG level), In(hsCRP level), In(ALT level), estimated
Urinary creatinine, age, sex, BMI, education, cigarette vs. Q1: OR $=1.30(1.03-1.64)$

Q3 vs. Q1: $\mathrm{OR}=1.09(0.86-1.39)$ 
Table 2 Summary of results in studies used as primary data: diabetes, prediabetes and hyperglycemia ( $n=12$ publications) (Continued)

\begin{tabular}{lll}
\hline & tolerance test or use of diabetes \\
& medication & \\
& & \\
Shankar \& Teppala & Diabetes: fasting serum glucose & BPA in quartiles $(\mathrm{ng} / \mathrm{mL}): \mathrm{Q} 1:<1.10 ;$ \\
2011 [42] & $>126 \mathrm{mg} / \mathrm{dL}$ or non-fasting serum & Q2: 1.10-2.10; Q3: $2.11-4.20 ; \mathrm{Q} 4:>4.20$ \\
& glucose $>200 \mathrm{mg} / \mathrm{dL}$ or HbA1c & \\
& $>6.5 \%$ or self-reported current use \\
& of oral hypoglycemic medication \\
& or insulin
\end{tabular}
$\begin{array}{ll}\text { Q4 vs. Q1: OR }=1.37(1.08-1.74) & \text { glomerular filtration rate, albumin level, total } \\ \text { p for trend not statistically significant } & \text { bilirubin level }\end{array}$

Q2 vs. Q1: OR $=1.42(1.03-1.96)$

Q3 vs. Q1: $\mathrm{OR}=1.48(1.05-2.08)$

Age, gender, race-ethnicity, education categories, smoking, alcohol intake, BMl, Q4 vs. Q1: OR = $1.68(1.22-2.30)$ systolic and diastolic blood pressure, urinary

$\mathrm{p}$ for trend $=0.002$

Normal weight participants:

Q4 vs. Q1: $\mathrm{OR}=3.17(1.23-8.18)$

Overweight/obese participants:

Q4 vs. Q1: OR = $1.56(1.09-2.24)$

Silver et al. 2011 [46] Type 2 diabetes: HbA1c $\geq 6.5 \%$ or BPA continuous (log-transformed) self-reported use of diabetes medication (insulin or blood sugar-lowering pills)

For a doubling in UBPA

concentration:

2003/04: $\mathrm{OR}=1.23(1.07-1.41)$

2005/06: OR $=1.06(0.95-1.19)$

2007/08: $O R=1.06(0.91-1.23)$

Pooled 2003/08: OR = $1.08(1.02$

1.16)

Incident diabetes (1 publication)

Sun et al. 2014 [61] Type 2 diabetes: self-reported

BPA in quartiles $(\mathrm{ng} / \mathrm{mL})$ :

NHS cohort / NHSIl cohor

diagnosis confirmed with one

of the ADA 1998 criteria: a)

elevated glucose concentration

and $\geq 1$ symptom related to

diabetes; b) no symptoms but

elevated glucose concentrations

on 2 separate occasions; or c)

treatment with insulin or ora

hypoglycemic medication
Q1: $<1.0 /<1.3$

Q2: 1.0-1.5/1.3-2.0

Q3: 1.5-2.7 / 2.0-3.5

Q4: $>2.7 />3.5$
NHS cohort (older women):

Q2 vs. Q1: $\mathrm{OR}=0.91(0.56-1.48)$

Q3 vs. Q1: $\mathrm{OR}=0.98(0.60-1.61)$

Q4 vs. Q1: OR $=0.81(0.48-1.38)$

$p$ for trend $=0.45$

NHSIl cohort (younger women):

Q2 vs. Q1: $\mathrm{OR}=1.34(0.70-2.27)$

Q3 vs. Q1: $\mathrm{OR}=1.91(1.11-3.29)$

Q4 vs. Q1: $\mathrm{OR}=2.08(1.17-3.69)$

$\mathrm{p}$ for trend $=0.02$

BPA in tertiles $(\mathrm{ng} / \mathrm{mL}): \mathrm{T} 1:<1.3$

T2: 1.3-3.2; T3: >3.2
T2 vs. $T 1: O R=1.42(1.14-1.76)$

$\mathrm{T} 3$ vs. $\mathrm{T} 1 \mathrm{O} O \mathrm{OR}=1.34(1.03-1.73)$

$\mathrm{p}$ for trend $=0.02$
Age, age ${ }^{2}$, urinary creatinine as natura

splines with $4^{\circ}$ of freedom, gender,

race-ethnicity, education,

household income, BMI, WC, smoking status

Prevalent prediabetes (1 publication)

[41]
Prediabetes: $\mathrm{FPG}=100-12$ $\mathrm{mg} / \mathrm{dL}$ or 2 -h glucose concentration $=140-199$
$\mathrm{mg} / \mathrm{dL}$ or $\mathrm{HbA} 1 \mathrm{c}=5.7-6.4 \%$ (ADA guidelines)
Age at urine sample collection, ethnicity, fasting status, time of sample collection, menopausal status, use of hormone replacement therapy (NHSII

only), urinary creatinine levels, smoking status, postmenopausal hormone use (NHS only), oral contraceptive use (NHSI only), physical activity, alcohol use, family history of diabetes, history of hypercholesterolemia or hypertension Alternative Health Eating Index score, BMI

Age, gender, race-ethnicity, education smoking, alcohol intake, BMI, physical inactivity, mean arterial blood pressure, C-reactive protein, total cholesterol/ HDL ratio 
Table 2 Summary of results in studies used as primary data: diabetes, prediabetes and hyperglycemia ( $n=12$ publications) (Continued)

\begin{tabular}{|c|c|c|c|c|}
\hline & & & $\begin{array}{l}\text { Stronger associations among women } \\
\text { and obese participants. }\end{array}$ & \\
\hline & & & Women: & \\
\hline & & & T2 vs. T1: OR = $1.36(0.96-1.91)$ & \\
\hline & & & T3 vs. T1: OR = $1.49(1.00-2.22)$ & \\
\hline & & & $p$ for trend $=0.04$ & \\
\hline & & & Obese: & \\
\hline & & & T2 vs. T1: OR = $1.71(1.05-2.80)$ & \\
\hline & & & T3 vs. T1: OR = 1.65 (1.04-2.80) & \\
\hline & & & $p$ for trend $=0.04$ & \\
\hline Prevalent hyperglyce & publications) & & & \\
\hline $\begin{array}{l}\text { Beydoun et al. } \\
2014 \text { [34] }\end{array}$ & $\begin{array}{l}\text { Hyperglycemia: FPG } \\
\geq 100 \mathrm{mg} / \mathrm{dL}\end{array}$ & BPA continuous (log-transformed) & $\mathrm{OR}=1.0(0.9-1.2)$ & $\begin{array}{l}\text { Age, sex, race, education, marital status, } \\
\text { smoking status, physical activity, dietary }\end{array}$ \\
\hline & & BPA in quartiles $(\mathrm{ng} / \mathrm{mL}): \mathrm{Q} 1: 0.3$ to & Q2 vs. Q1: OR = $0.9(0.52-1.42)$ & $\begin{array}{l}\text { energy intake, urinary creatinine, survey } \\
\text { wave }\end{array}$ \\
\hline & & $\begin{array}{l}<1.0 ; \text { Q2: } 1.0 \text { to }<2.0 ; \text { Q3: } 2.0 \text { to } \\
<3.7 ; \text { Q4: } \geq 3.7\end{array}$ & Q3 vs. Q1: $\mathrm{OR}=1.2(0.7-1.9)$ & \\
\hline & & & Q4 vs. Q1: OR = $1.1(0.6-1.9)$ & \\
\hline & & & $p$ for trend $=0.55$ & \\
\hline & & $\begin{array}{l}\text { Ratio of BPA-to-creatinine continuous } \\
\text { (log-transformed) }\end{array}$ & $\mathrm{OR}=1.0(0.9-1.2)$ & \\
\hline & & Ratio of BPA-to-creatinine in quartiles: & Q2 vs. Q1: $\mathrm{OR}=1.1(0.8-1.6)$ & \\
\hline & & $\begin{array}{l}\text { Q1: } 0.001 \text { to }<0.01 ; \mathrm{Q} 2: 0.01 \text { to }<0.02 \text {; } \\
\text { Q3: } 0.02 \text { to }<0.03 ; \mathrm{Q} 4: \geq 0.03\end{array}$ & Q3 vs. Q1: $\mathrm{OR}=1.3(0.8-1.9)$ & \\
\hline & & & Q4 vs. Q1: OR = $1.2(0.8-1.9)$ & \\
\hline & & & $p$ for trend $=0.30$ & \\
\hline Eng et al. 2013 [38] & Abnormal glucose: FPG & BPA in quartiles $(\mathrm{ng} / \mathrm{mL})$ & & Age, gender, race/ethnicity, urine \\
\hline & $\geq 10$ & Q1: $<1.3$ & Q2 vs. Q1: OR = $0.77(0.33-1.78)$ & $\begin{array}{l}\text { creatinine, poverty-to-income ratio, } \\
\text { serum cotinine as a marker of smoking }\end{array}$ \\
\hline & & Q2: $1.3-2.6$ & Q3 vs. Q1: OR = $1.32(0.57-3.04)$ & status, soda consumption, BMI percentile \\
\hline & & Q3: 2.6-4.9 & Q4 vs. Q1: OR = $0.63(0.22-1.82)$ & \\
\hline & & Q4: $>4.9$ & & \\
\hline
\end{tabular}

ADA American Diabetes Association; ALT alanine aminotransferase; BMI body mass index; BPA bisphenol A; FPG fasting plasma glucose; HbA1c glycated hemoglobin; HDL high density lipoprotein; hsCRP high sensitivity C-reactive protein; NHS Nurses' Health Study; OR odds ratio, SD standard deviation; T2D type 2 diabetes; TG triglycerides; uBPA urinary bisphenol A; WC waist circumference 
Table 3 Summary of results in studies used as primary data: indicators of anthropometry and adiposity ( $\mathrm{n}=16$ publications)

Reference $\quad$ Outcomes \& definitions used $\quad$ Urinary BPA categorisation $\quad$ Results

\section{In children}

Prevalent overweight (4 publications)

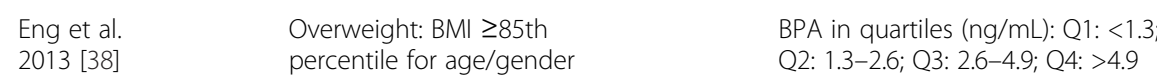

\section{Harley et al.}

$2013[64]$

Overweight: BMI $\geq 85$ th

percentile at 5 and 9 years

of age

$\begin{array}{ll}\text { Li et al. } 2013 & \text { Overweight: age- and } \\ \text { [49] } & \text { gender-specific weight } \\ & \text { >90th percentile of the } \\ & \text { underlying population }\end{array}$

$\begin{array}{ll}\text { Trasande } & \text { Overweight: BMI z-score } \\ \text { et al. 2012 } & \geq 1.036 \text { (85th percentile for } \\ \text { [47] } & \text { age/sex) }\end{array}$

Prevalent obesity (3 publications)

$\begin{array}{ll}\text { Bhandari et al. } & \text { Obesity: BMI } \geq 95 \text { th percentile } \\ 2013[35] & \text { for age/gender }\end{array}$ Q2: 1.5-2.7; Q3: 2.8-5.4; Q4: > 5.4
BPA continuous (log-transformed)

BPA in quartiles (ng/mL): Q1: $<1.5$

Q2 vs. Q1: $\mathrm{OR}=1.00(0.74-1.36)$ $\mathrm{Q} 3$ vs. $\mathrm{Q} 1: \mathrm{OR}=1.17(0.89-1.54)$ Q4 vs. Q1: $\mathrm{OR}=1.07(0.80-1.44)$ $\mathrm{OR}=1.07(0.90-1.28)$

Ratio of BPA-to-creatinine level as 5 years

Ratio of BPA-to-creatinine level in 3 tertiles at 5 years $(\mu \mathrm{g} / \mathrm{g})$ :

T1: <LOD-2.4; T2: 2.4-4.5 $\mu \mathrm{g} / \mathrm{g}$; T3: $4.6-349.8 \mu \mathrm{g} / \mathrm{g}$

Ratio of BPA-to-creatinine level as continuous ( $\log _{2}$ - transformed) at 9 years

Ratio of BPA-to-creatinine level in 3 groups at 9 years $(\mu \mathrm{g} / \mathrm{g})$ :

G1: <LOD (<0.4); G2: detectable $<$ median (0.4-1.8); G3: detectable > median (1.8-22.5)

BPA in 2 classes $(\mathrm{ng} / \mathrm{mL})$ : Low BPA level: $<2$ (reference); high BPA level: $\geq 2(2 \mathrm{mg} / \mathrm{L}$ is about the median urine BPA level in the U.S. population)

BPA continuous (log-transformed) BPA in quartiles (ng/mL): Q1: $\leq 1.5$ Q2: 1.5-2.7; Q3: 2.8-5.5; Q4: > 5.6

T2 vs. T1: $\mathrm{OR}=0.80(0.45-1.42)$

T3 vs. $\mathrm{T} 1: \mathrm{OR}=1.36(0.75-2.47)$

$\mathrm{OR}=1.06(0.85-1.33)$

G2 vs. G1: $\mathrm{OR}=3.08(1.18-8.02)$

G3 vs. G1: OR $=4.20(1.60-11.02)$

Girls

All: $\mathrm{OR}=1.29(0.83-2.01)$

Age 9-12: $O R=2.32(1.15-4.65)$

Age $>12: \mathrm{OR}=0.90(0.48-1.72)$

Boys

All: $\mathrm{OR}=0.82(0.55-1.23)$

Age 9-12: $\mathrm{OR}=0.71(0.34-1.45)$

Age $>12: \mathrm{OR}=0.87(0.52-1.45)$

$\mathrm{OR}=1.04(0.92-1.18)$

Q2 vs. Q1: $\mathrm{OR}=1.26(0.96-1.64)$

Q3 vs. Q1: $\mathrm{OR}=1.28(0.98-1.66)$

Q4 vs. Q1: $\mathrm{OR}=1.26(0.86-1.82)$

$\mathrm{OR}=1.25(1.09-1.43)$

Q2 vs. Q1: $\mathrm{OR}=2.35(1.56-3.53)$
Age, gender, race/ethnicity, urine creatinine, poverty-to-income ratio, serum cotinine as a marker of smoking status, soda consumption

Maternal prepregnancy BMI, household income, maternal education level, maternal years of residence in the United States, child's environmental tobacco smoke exposure, soda intake, fast food intake, and sweet consumption

Age, gender, school, residence, paternal and maternal education and overweight, playing video games, unbalanced diet, eating junk food, vegetables or fruit, depression scores, sports/activities

Sex, caloric intake, television watching, poverty to income ratio, parental education, serum cotinine level, urinary creatinine level, age, race/ethnicity categories

Age, sex, race/ethnicity, education, moderate activity, urinary creatinine, serum cotinine 
Table 3 Summary of results in studies used as primary data: indicators of anthropometry and adiposity ( $\mathrm{n}=16$ publications) (Continued)

\begin{tabular}{|c|c|c|}
\hline $\begin{array}{l}\text { Eng et al. } \\
2013 \text { [38] }\end{array}$ & $\begin{array}{l}\text { Obesity: BMI } \geq 95 \text { th percentile } \\
\text { for age/gender }\end{array}$ & $\begin{array}{l}\text { BPA in quartiles }(\mathrm{ng} / \mathrm{mL}): \mathrm{Q} 1:<1.3 \\
\text { Q2: 1.3-2.6; Q3: 2.6-4.9; Q4: >4.9 }\end{array}$ \\
\hline \multirow{2}{*}{$\begin{array}{l}\text { Trasande } \\
\text { et al. } 2012 \\
\text { [47] }\end{array}$} & \multirow{2}{*}{$\begin{array}{l}\text { Obesity: BMI } z \text {-score } \geq 1.64 \\
\text { (95th percentile for age/sex) }\end{array}$} & BPA continuous (log-transformed) \\
\hline & & $\begin{array}{l}\text { BPA in quartiles }(\mathrm{ng} / \mathrm{mL}): \mathrm{Q} 1: \leq 1.5 \\
\text { Q2: 1.5-2.7; Q3: 2.8-5.5; Q4: >5.6 }\end{array}$ \\
\hline
\end{tabular}

Prevalent elevated waist circumference

$\begin{array}{ll}\text { Eng et al. } & \text { Abnormal WC: WC } \geq 90 \text { th } \\ 2013[38] & \text { percentile for age/gender } \\ & \text { Abnormal WC-to-height ratio: } \\ & \text { WC-to-height ratio } \geq 0.5\end{array}$

Other

Braun et al. Change in BMl z-score between 2014 [63] 2 and 5 years of age, as continuous

Eng et al.

2013 [38]

Harley et al.

2013 [64]
BPA in quartiles $(\mathrm{ng} / \mathrm{mL}): 01:<1.3$ Q2: 1.3-2.6; Q3: 2.6-4.9; Q4: >4.9 Abnormal WC-to-height
Ratio of BPA-to-creatinine continuous ( $\log _{10}$-transformed)

Ratio of BPA-to-creatinine in tertiles ( $\mu \mathrm{g} / \mathrm{g}$ creatinine):

T1: 2.1-11; T2: 11-20; T3: 20-314

BPA in quartiles $(\mathrm{ng} / \mathrm{mL})$ : Q1: $<1.3$; Q2: 1.3-2.6: Q3: 2.6-4.9: Q4: >4.9

Ratio of BPA-to-creatinine level at 5 years as continuous $\left(\log _{2^{-}}\right.$
Q3 vs. Q1: $\mathrm{OR}=1.78(1.13-2.79)$

Q4 vs. Q1: $\mathrm{OR}=2.55(1.65-3.95)$

$\mathrm{p}$ for trend $=0.002$

Stratified analyses by sex ( $p$ for interaction $=0.07$ ): association of strong magnitude and statistically significant among boys transformed)

Q3 vs. Q1: $\mathrm{OR}=1.41(1.07-1.87)$ body fat $\geq 85$ th percentile for

Incident overweight: BMI $\geq 85$ th percentile at 9 years of age
Q2 vs. Q1: $\mathrm{OR}=1.73(1.16-2.58)$ Q3 vs. Q1: $\mathrm{OR}=1.63(1.08-2.46)$ Q4 vs. Q1: $\mathrm{OR}=2.05(1.38-3.04)$ Continuous: $\mathrm{OR}=1.24(1.08-1.44)$ Q2 vs. Q1: $\mathrm{OR}=2.24(1.54-3.24)$ Q3 vs. Q1: $\mathrm{OR}=2.08(1.46-2.96)$ Q4 vs. Q1: $\mathrm{OR}=2.57(1.72-3.83)$

Age, gender, race/ethnicity, urine creatinine, poverty-to-income ratio, serum cotinine as marker of smoking status, soda consumption

Sex, caloric intake, television watching, poverty-to-income ratio, parental education serum cotinine level, urinary creatinine level, age, race/ethnicity categories

Abnormal WC

Q2 vs. Q1: $\mathrm{OR}=1.33(0.90-1.97)$

Q3 vs. Q1: $\mathrm{OR}=1.16(0.75-1.81)$

Q4 vs. Q1: $\mathrm{OR}=1.40(0.91-2.15)$

Abnormal WC-to-height ratio

Q2 vs. Q1: OR = $1.37(0.97-1.92)$

Q4 vs. Q1: $\mathrm{OR}=1.56(1.11-2.17)$

Per 10 -fold increase: $\beta=-0.2$ $(-0.6,0.1)$

T2 vs. T1: $\beta=0.0(-0.3,0.3)$

T3 vs. T1: $\beta=-0.2(-0.5,0.1)$

Q2 vs. Q1: $\mathrm{OR}=4.85(0.80-21.4)$

Q3 vs. Q1: $\mathrm{OR}=5.36(0.71-43.3)$

$\mathrm{Q} 4$ vs. Q1: $\mathrm{OR}=2.10(0.24-17.8)$

$\mathrm{OR}=1.02(0.84-1.23)$
Age, gender, race/ethnicity, urine creatinine, poverty-to-income ratio, serum cotinine as a marker of smoking status, soda consumption

Maternal race, marital status, parity, age at delivery, household income, education, employment, insurance, BMl at 16 weeks of pregnancy, depressive symptoms at baseline, prenatal serum cotinine

Age, gender, race/ethnicity, urine creatinine, poverty-to-income ratio, serum cotinine as a marker of smoking status, soda consumption

Maternal prepregnancy BMI, household income, maternal education level, maternal years of residence in the United States, child's environmental tobacco smoke exposure, soda 
Table 3 Summary of results in studies used as primary data: indicators of anthropometry and adiposity ( $n=16$ publications) (Continued)

\begin{tabular}{|c|c|c|}
\hline & & $\begin{array}{l}\text { Ratio of BPA-to-creatinine level at } \\
5 \text { years in tertiles }(\mu \mathrm{g} / \mathrm{g}) \text { : }\end{array}$ \\
\hline & & $\begin{array}{l}\text { T1: <LOD-2.4; T2: 2.4-4.5; } \\
\text { T3: 4.6-349.8 }\end{array}$ \\
\hline $\begin{array}{l}\text { Wang et al. } \\
2012 \mathrm{~b}[50]\end{array}$ & Prevalent BMl as continuous $\left(\mathrm{kg} / \mathrm{m}^{2}\right)$ & $\begin{array}{l}\text { BPA continuous (log-transformed } \\
\text { and corrected for specific gravity) }\end{array}$ \\
\hline $\begin{array}{l}\text { Wells et al. } \\
2013 \text { [48] }\end{array}$ & $\begin{array}{l}\text { Prevalent waist-to-height } \\
\text { ratio as continuous }\end{array}$ & $\begin{array}{l}\text { BPA in quartiles }(\mathrm{ng} / \mathrm{mL}): \text { Q1: }<1.2 ; \\
\text { Q2: 1.2-2.6; Q3: } 2.6-5.1 ; \mathrm{Q} 4:>5.1\end{array}$ \\
\hline
\end{tabular}

In adults

Prevalent overweight (3 publications)

\begin{tabular}{|c|c|}
\hline $\begin{array}{l}\text { Carwile \& } \\
\text { Michels } 2011 \\
\text { [36] }\end{array}$ & $\begin{array}{l}\text { Overweight: } 25 \leq \mathrm{BMI}< \\
30 \mathrm{~kg} / \mathrm{m}^{2} \text { (reference: BMl } \\
<25 \mathrm{~kg} / \mathrm{m}^{2} \text { ) }\end{array}$ \\
\hline $\begin{array}{l}\text { Kim et al. } \\
2011 \text { [55] }\end{array}$ & $\begin{array}{l}\text { Overweight: BMI = } 23-24.9 \\
\mathrm{~kg} / \mathrm{m}^{2} \text {, according to the WHO } \\
\text { definitions for the Asian } \\
\text { populations (reference: BMI } \\
<18.5 \mathrm{~kg} / \mathrm{m}^{2} \text { ) }\end{array}$ \\
\hline $\begin{array}{l}\text { Wang et al. } \\
\text { 2012a [52] }\end{array}$ & $\begin{array}{l}\text { Generalized overweight: } 24 \\
\leq \mathrm{BMl}<28 \mathrm{~kg} / \mathrm{m}^{2} \text {, according } \\
\text { to Chinese criteria (reference = } \\
\mathrm{BMl}<24 \mathrm{~kg} / \mathrm{m}^{2} \text { ) }\end{array}$ \\
\hline
\end{tabular}

Prevalent obesity (4 publications)

$\begin{array}{ll}\begin{array}{l}\text { Carwile \& } \\ \text { Michels 2011 } \\ \text { [36] }\end{array} & \begin{array}{l}\text { Obesity: BMI } \geq 30 \mathrm{~kg} / \mathrm{m}^{2} \\ \text { (reference: } \mathrm{BMl}<25 \mathrm{~kg} / \mathrm{m}^{2} \text { ) }\end{array} \\ \begin{array}{ll}\text { Kim et al. } \\ 2011[55]\end{array} & \begin{array}{l}\text { Obesity: BMI } \geq 25 \mathrm{~kg} / \mathrm{m}^{2}, \\ \text { according to the WHO } \\ \text { definitions for the Asian } \\ \text { populations (reference }= \\ \text { BMl }<18.5 \mathrm{~kg} / \mathrm{m}^{2} \text { ) }\end{array} \\ & \begin{array}{l}\text { Obesity: } \mathrm{BMl} \geq 30 \mathrm{~kg} / \mathrm{m}^{2} \\ \text { (reference }=\mathrm{BMl}<30 \mathrm{~kg} / \mathrm{m}^{2} \text { ) }\end{array} \\ 2012 \text { [44] } & \end{array}$
boys but not girls.

BPA in quartiles $(\mathrm{ng} / \mathrm{mL}): \mathrm{Q} 1: \leq 1.1$ Q2: 1.2-2.3; Q3: 2.4-4.6; Q4: $\geq 4.7$

BPA continuous (log-transformed)

BPA in quartiles $(\mathrm{ng} / \mathrm{mL}): \mathrm{Q} 1: \leq 0.47$; Q2: 0.48-0.81; Q3: 0.82-1.43: Q4: $>1.43$

BPA in quartiles $(\mathrm{ng} / \mathrm{mL}): 01: \leq 1.1$ Q2: 1.2-2.3; Q3: 2.4-4.6; Q4: $\geq 4.7$

BPA continuous (log-transformed)

BPA in quartiles ( $\mathrm{ng} / \mathrm{mL}): \mathrm{Q} 1:<1.10$; Q2: 1.10-2.10; Q3: 2.11-4.20; Q4: >4.20
T2 vs. T1: 0.91 (0.48-1.73)

T3 vs. T1: $1.28(0.65-2.51)$

$\beta=0.017(0.002-0.032)$

Change in waist-to-height ratio: Q2 vs. Q1: $\beta=0.011(0.001-0.020)$ Q3 vs. Q1: $\beta=0.010(0.001-0.019)$ Q4 vs. Q1: $\beta=0.016(0.007-0.026)$ Significant associations among

intake, fast food intake, and sweet consumption at age 5 years

Age, sex

Urinary creatinine, age, sex, race/ethnicity, education, smoking status based on serum cotinine, caloric intake

Q2 vs. Q1: $\mathrm{OR}=1.66(1.21-2.27)$ $\mathrm{Q} 3$ vs. $\mathrm{Q} 1: \mathrm{OR}=1.26(0.85-1.87)$ Q4 vs. Q1: $\mathrm{OR}=1.31(0.80-2.14)$ Adjusted proportional change $(95 \% \mathrm{Cl})=1.01(0.78-1.31)$

Q2 vs. Q1: $\mathrm{OR}=1.23(0.97-1.57)$ $\mathrm{Q} 3$ vs. $\mathrm{Q} 1: \mathrm{OR}=1.28(1.01-1.63)$ Q4 vs. Q1: $\mathrm{OR}=1.24(0.97-1.59)$

Q2 vs. Q1: $\mathrm{OR}=1.85(1.22-2.79)$ Q3 vs. Q1: $\mathrm{OR}=1.60(1.05-2.44)$ Q4 vs. Q1: OR = $1.76(1.06-2.94)$ Adjusted proportional change $(95 \% \mathrm{Cl})=0.96(0.75-1.23)$

Q2 vs. Q1: $\mathrm{OR}=1.40(1.10-1.76)$ $\mathrm{Q} 3$ vs. $\mathrm{Q} 1: \mathrm{OR}=1.59(1.25-2.02)$
Age, gender, race/ethnicity, education, smoking status, urinary creatinine

Age, gender, education, income, cigarette smoking status, place of residence, urinary creatinine

Age, sex, urinary creatinine, smoking, alcohol drinking, education levels, systolic blood pressure, HDL cholesterol, LDL cholesterol, total cholesterol, TG, hsCRP, fasting plasma glucose, fasting serum insulin, serum ALT and GGT

Age, gender, race/ethnicity, education, smoking status, urinary creatinine

Age, gender, education, income, cigarette smoking status, place of residence, urinary creatinine

Age, gender, race/ethnicity, education categories, smoking, alcohol consumption, 
Table 3 Summary of results in studies used as primary data: indicators of anthropometry and adiposity ( $\mathrm{n}=16$ publications) (Continued)

\begin{tabular}{ll}
\hline & \\
Wang et al. & Generalized obesity: BMl $\geq 28$ \\
2012a [52] & $\mathrm{kg} / \mathrm{m}^{2}$, according to Chinese \\
& criteria (reference: $\mathrm{BMl}<28$ \\
$\left.\mathrm{~kg} / \mathrm{m}^{2}\right)$
\end{tabular}

Prevalent elevated waist circumference

(4 publications)

$\begin{array}{ll}\text { Carwile \& } & \text { Elevated WC: } W C \geq 102 \mathrm{~cm} \\ \text { Michels } 2011 & \text { in men and WC } \geq 88 \mathrm{~cm} \text { in }\end{array}$

[36]

women

$\begin{array}{ll}\text { Ko et al. } 2014 & \text { Abdominal obesity: WC } \\ {[57]} & \geq 90 \mathrm{~cm} \text { in men and WC } \\ & \geq 85 \mathrm{~cm} \text { in women }\end{array}$

$\begin{array}{ll}\text { Shankar et al. } & \text { Abdominal obesity: WC } \\ \text { 2012 [44] } & \geq 102 \mathrm{~cm} \text { in men and WC } \\ & \geq 88 \mathrm{~cm} \text { in women }\end{array}$

Wang et al.

2012a [52]

Other

Galloway

et al. 20

[58]

Kim et al.

2011 [55]
Abdominal obesity: WC $\geq 90 \mathrm{~cm}$ in men and WC $\geq 85 \mathrm{~cm}$ in women

Prevalent WC as continuous

Prevalent weight as continuous

Prevalent normal weight: $\mathrm{BMI}=18.5-22.9 \mathrm{~kg} / \mathrm{m}^{2}$, according to the WHO definitions for the Asian
BPA in quartiles $(\mathrm{ng} / \mathrm{mL}): 01: \leq 0.47$ Q2: 0.48-0.81: Q3: 0.82-1.43: Q4: $>1.43$

BPA in quartiles (ng/mL): Q1: $\leq 1.1$; Q2: 1.2-2.3; Q3: 2.4-4.6; Q4: $\geq 4.7$

BPA in quartiles $(\mu \mathrm{g} / \mathrm{mL})$

Q1: <0.853; Q2: 0.853-1.407;

Q3: 1.407-2.594; Q4: > 2.594

BPA in quartiles $(\mathrm{ng} / \mathrm{mL})$ :

Q1: <1.10; Q2: 1.10-2.10; Q3: 2.114.20; Q4: >4.20

BPA in quartiles $(\mathrm{ng} / \mathrm{mL}): \mathrm{Q} 1: \leq 0.47$ Q2: 0.48-0.81: Q3: 0.82-1.43: Q4: $>1.43$

Daily BPA excretion ( $\mu \mathrm{g} /$ day) as a continuous variable

BPA continuous (log-transformed)
Q4 vs. Q1: OR $=1.69(1.30-220)$ p for trend $<0.0001$

Associations still significant in analyses stratified by sex.

Q2 vs. Q1: OR = $1.14(0.87-1.50)$ $\mathrm{Q} 3$ vs. $\mathrm{Q} 1: \mathrm{OR}=1.19(0.90-1.57)$

Q4 vs. Q1: OR = $1.50(1.15-1.97)$

Q2 vs. Q1: $\mathrm{OR}=1.62(1.11-2.36)$ Q3 vs. Q1: $O R=1.39(1.02-1.90)$ Q4 vs. Q4: $\mathrm{OR}=1.58(1.03-2.42)$ Q2 vs. Q1: $1.117(0.757-1.649)$ Q3 vs. Q1: $1.337(0.908-1.967)$ Q4 vs. Q1: 1.938 (1.314-2.857) $\mathrm{p}$ for trend $=0.01$

Q2 vs. Q1: $\mathrm{OR}=1.63(1.20-2.22)$ $\mathrm{Q} 3$ vs. $\mathrm{Q} 1: \mathrm{OR}=1.66(1.28-2.14)$ Q4 vs. Q1: $\mathrm{OR}=1.59(1.21-2.09)$ p for trend $=0.0009$

Associations still significant in analyses stratified by sex

Q2 vs. Q1: $\mathrm{OR}=1.26(1.02-1.57)$ $\mathrm{Q} 3$ vs. $\mathrm{Q} 1: \mathrm{OR}=1.28(1.03-1.59)$ Q4 vs. Q1: $\mathrm{OR}=1.28(1.03-1.60)$

WC: $\beta=0.0062(0.0016-0.0108)$ Weight: $\beta=0.0064(0.0023-0.0104)$

Adjusted proportional change $(95 \% \mathrm{Cl})=0.92(0.72-1.17)$ physical inactivity, diabetes, hypertension total cholesterol

Age, sex, urinary creatinine, smoking, alcoho drinking, education levels, systolic blood pressure, HDL cholesterol, LDL cholesterol, total cholesterol, TG, hsCRP, fasting plasma glucose, fasting serum insulin, serum ALT and GGT

Age, gender, race/ethnicity, education, smoking status, urinary creatinine

Age, sex, urinary creatinine, education, income, alcohol consumption, smoking status

Age, gender, race/ethnicity, education categories, smoking, alcohol consumption, physical inactivity, diabetes, hypertension, total cholestero

Age, sex, urinary creatinine, smoking, alcoho drinking, education levels, systolic blood pressure, HDL cholesterol, LDL cholesterol, total cholesterol, TG, hsCRP, fasting plasma glucose, fasting serum insulin, serum ALT and GGT

Age, sex, study site

Age, gender, education, income, cigarette smoking status, place of residence, urinary creatinine 
Table 3 Summary of results in studies used as primary data: indicators of anthropometry and adiposity ( $n=16$ publications) (Continued)

\begin{tabular}{|c|c|c|c|c|}
\hline & $\begin{array}{l}\text { populations (reference: BMI } \\
<18.5 \mathrm{~kg} / \mathrm{m}^{2} \text { ) }\end{array}$ & & & \\
\hline \multirow{5}{*}{$\begin{array}{l}\text { Song et al. } \\
2014 \text { [62] }\end{array}$} & \multirow{5}{*}{$\begin{array}{l}\text { Weight change rate (WCR) } \\
\text { during follow-up (kg/year) }\end{array}$} & BPA in quartiles $(\mathrm{nmol} / \mathrm{L})$ : & & \multirow{5}{*}{$\begin{array}{l}\text { Age at baseline, urinary creatinine } \\
\text { concentration, cohort origin, menopausal } \\
\text { status, smoking, alcohol consumption, } \\
\text { physical activity, alternative healthy eating } \\
\text { index, total energy intake }\end{array}$} \\
\hline & & Q1: median (IQR) = $3.6(2.6-4.5)$ & Q2 vs. Q1: WCR $=0.15(0.00-0.31)$ & \\
\hline & & Q2: median (IQR) = 6.4 (5.8-7.3) & Q3 vs. Q1: WCR $=0.18(0.03-0.34)$ & \\
\hline & & Q3: median (IQR) $=10.5(9.0-12.1)$ & Q4 vs. Q1: WCR $=0.23(0.07-0.38)$ & \\
\hline & & Q4: median $(\mathrm{IQR})=21.9(16.8-35.7)$ & $p$ for trend $=0.02$ & \\
\hline \multirow{8}{*}{$\begin{array}{l}\text { Zhao et al. } \\
2012 \text { [53] }\end{array}$} & \multirow{8}{*}{$\begin{array}{l}\text { Fat mass, fat-free mass, body weight, BMI, } \\
\text { WC, hip circumference, waist-hip ratio (all } \\
\text { variables as continuous) }\end{array}$} & \multirow[t]{8}{*}{ BPA continuous } & Fat mass: $r=0.35(p<0.001)$ & \multirow[t]{8}{*}{ Age } \\
\hline & & & Fat-free mass: $r=0.186(p=0.009)$ & \\
\hline & & & Body weight: $r=0.24(p=0.001)$ & \\
\hline & & & BMI: $r=0.298(p<0.001)$ & \\
\hline & & & $W C: r=0.296(p<0.001)$ & \\
\hline & & & $\begin{array}{l}\text { Hip circumference: } r=0.27 \\
(p<0.001)\end{array}$ & \\
\hline & & & $\begin{array}{l}\text { Waist-hip ratio: } r=0.149 \\
(p=0.035)\end{array}$ & \\
\hline & & & $\begin{array}{l}\text { With additional adjustment for age } \\
\text { and BMI, BPA was still significantly } \\
\text { associated with fat mass }(r=0.193 \text {, } \\
p=0.006) \text { but not with fat-free mass. }\end{array}$ & \\
\hline
\end{tabular}

ALT alanine aminotransferase; $B M I$ body mass index; BPA bisphenol A; GGT gamma glutamyltransferase; HDL high density lipoprotein; $h s C R P$ high sensitivity C-reactive protein; IQR interquartile range; $L D L$ low density lipoprotein; $L O D$ limit of detection; $O R$ odds ratio; TG triglycerides; WC waist circumference; WCR weight change rate 
Table 4 Summary of results in studies used as primary data: cardiovascular disease and hypertension ( $n=9$ publications)

Reference Outcomes \& definitions used Urinary BPA categorisation Results

Prevalent CVD (5 publications)

Casey \&

Neidell 2013

[37]

CHD: self-report of doctor diagnosis

BPA continuous (not log-

transformed)

BPA continuous (log-transformed)

Per 10-fold increase:

BPA in quartiles $(\mathrm{ng} / \mathrm{mL})$

Q1: $<1.2 ;$ Q2: 1.2-2.2; Q3: 2.3-4.2;

Q4: $>4.2$

Lakind et al. CHD: self-report of doctor diagnosis BPA continuous 2012 [33] Heart attack: self-report of doctor diagnosis

Lang et al. 2008 [39]

Heart attack, angina, CHD, CVD (any BPA continuous

diagnoses of $\mathrm{Ml}$, angina or $\mathrm{CHD}$,

stroke; all self-reported doctor

diagnoses
2003/04: OR $=1.584(1.066-2.354)$

2005/06: OR $=1.178(0.765-1.815)$

2007/08: OR $=1.649(1.025-2.654)$

Pooled 2003/08: OR = 1.280 (0.993-1.649)

Adjustment in model(s) used for review

Age, sex, urinary creatinine concentration, race/ethnicity, income, smoking, body mass index, waist circumference, veteran/military status, citizenship status, marital status, household size, pregnancy status, language at subject interview, health insurance coverage, employment status in the prior week, consumption of bottled water in the past $24 \mathrm{~h}$, consumption of alcohol, annual consumption of tuna fish, presence of emotional support in one's life, being on a diet, using a water treatment device, access to a routine source of health care, vaccinated for Hepatitis A or B, consumption of dietary supplements (vitamins or minerals), inability to purchase balanced meals on a consistent basis + survey cycle for pooled analyses

Pooled 2003/08:

Q2 vs. Q1: $0.520(0.250-1.084)$ Q3 vs. Q1: $1.006(0.508-1.994)$ Q4 vs. Q1: $1.520(0.774-2.987)$

$\mathrm{CHD}$, per unit increase:

2003/04: OR = $1.03(0.978-1.09)$

2005/06: OR $=1.02(0.996-1.04)$

2007/08: OR $=0.996(0.951-1.04)$

2009/10: OR $=1.00(0.998-1.01)$

Pooled 2003/10: OR = 1.004 (0.998-1.009)

Heart attack, per unit increase:

2003/04: OR = $1.04(0.996-1.09)$

2005/06: OR $=1.02(0.996-1.04)$

2007/08: $O R=0.987(0.941-1.04)$

2009/10: OR = $1.00(0.999-1.01)$

Pooled 2003/10: OR = 1.002 (0.998-1.007)

Per SD increase:

Heart attack: $\mathrm{OR}=1.40(1.11-1.78)$,

Angina: $\mathrm{OR}=1.28(1.09-1.50)$

CHD: $\mathrm{OR}=1.63(1.18-2.26)$

CVD: $\mathrm{OR}=1.39(1.18-1.63)$

Stroke: $\mathrm{OR}=0.97(0.74-1.27)$
Age, gender, ethnicity, education, income, smoking, heavy drinking, BMI, waist circumference, energy intake, family history of heart attack, hypertension, sedentary activity, total cholesterol, urinary creatinine. Pooled 2003/10 models were further adjusted for survey cycle, but were not adjusted for energy intake and sedentary activity.

Age, gender, race/ethnicity, education, income, BMI, WC, smoking status, urinary creatinine 
Table 4 Summary of results in studies used as primary data: cardiovascular disease and hypertension ( $n=9$ publications) (Continued)

Melzer et al. $\quad \mathrm{Ml}$, angina, CHD, CVD (any BPA continuous

2010 [40] diagnoses of Ml, angina or CHD)

all self-reported doctor diagnoses

\section{Melzer et al. CAD: no, intermediate, severe}

$2012 b[59]$

(assessed by angiography)

Incident CVD (1 publication)

Melzer et al. Incident CAD during follow-up:

2012a [60] recorded hospital admission and/or

died with CAD as underlying cause

\section{Prevalent hypertension (3 studies)}

Bae et al. 2012 Hypertension: systolic BP $\geq 140 \mathrm{~mm}$ [54] $\mathrm{Hg}$ or diastolic $\mathrm{BP} \geq 90 \mathrm{~mm} \mathrm{Hg}$

BPA continuous

BPA continuous

BPA continuous
$\mathrm{Ml}$, per SD increase:

2003/04: OR $=1.40(1.07-1.84)$

2005/06: OR $=1.39(1.00-1.94)$

Pooled 2003/06: OR = $1.32(1.15-1.52)$

Angina, per SD increase:

2003/04: OR $=1.27(1.06-1.54)$

2005/06: OR $=1.16(0.88-1.53)$

Pooled 2003/06: OR = 1.24 (1.07-1.43)

CHD, per SD increase:

2003/04: OR $=1.60(1.11-2.32)$

2005/06: OR $=1.33(1.01-1.75)$

Pooled 2003/06: OR = $1.42(1.17-1.72)$

$C V D$, per SD increase:

2003/04: OR = $1.34(1.10-1.66)$

2005/06: OR $=1.18(0.88-1.59)$

Pooled 2003/06: OR = $1.26(1.11-1.44)$

Per SD increase:

Severe vs. no CAD: $\mathrm{OR}=1.43(1.03-1.98)$

\section{Per SD increase:}

$\mathrm{OR}=1.11(1.00-1.23)$

All participants:

Ratio of BPA-to-creatinine in quartiles ( $\mu \mathrm{g} / \mathrm{g}$ creatinine): Q1: $<0.37$; Q2: 0.37-0.73; Q3: 0.73-1.33; Q4: $>1.33$

Q2 vs. Q1: OR $=1.21(0.80-1.84)$

$\mathrm{Q} 3$ vs. Q1: $\mathrm{OR}=1.16(0.78-1.72)$
Intermediate vs. no CAD: $\mathrm{OR}=1.69(0.98-2.94)$ status

Age, gender, ethnicity, education, income, BMI, WC,

smoking status, urinary creatinine

Q4 vs. Q1: OR = $1.27(0.85-1.88)$

Stratification by gender: non-significant results in males and females.

Significant associations in participants without previous history of hypertension

Q2 vs. Q1: $\mathrm{OR}=2.23(1.21-4.12)$

Q3 vs. Q1: $\mathrm{OR}=1.79(1.01-3.17)$
Age, sex, urinary creatinine, education level, occupational group, BMI, cigarette smoking, average of the 2 systolic BP readings, total cholesterol, LDL cholesterol, HDL cholesterol, TG, level of physical activity

Age, sex, height, weight, date of examination, mean fasting blood glucose, smoking status, current consumption of alcohol 
Table 4 Summary of results in studies used as primary data: cardiovascular disease and hypertension ( $n=9$ publications) (Continued)

\begin{tabular}{|c|c|c|c|c|}
\hline & & & Q4 vs. Q1: OR = $2.35(1.33-4.17)$ & \\
\hline \multirow{4}{*}{$\begin{array}{l}\text { Shankar \& } \\
\text { Teppala } 2012 \\
\text { [43] }\end{array}$} & \multirow{4}{*}{$\begin{array}{l}\text { Hypertension: current blood- } \\
\text { pressure-reducing medication use } \\
\text { and/or systolic BP }>140 \mathrm{mmHg} \text { and/ } \\
\text { or diastolic BP }>90 \mathrm{~mm} \mathrm{Hg}\end{array}$} & BPA continuous (log-transformed) & $\mathrm{OR}=1.11(1.01-1.22)$ & \multirow{4}{*}{$\begin{array}{l}\text { Age, sex, race/ethnicity, education categories, smoking, } \\
\text { alcohol intake, BMI, diabetes, total cholesterol }\end{array}$} \\
\hline & & \multirow{3}{*}{$\begin{array}{l}\text { BPA in tertiles }(\mathrm{ng} / \mathrm{mL}): \mathrm{T} 1:<1.5 ; \\
\text { T2: } 1.5-4.0 ; \mathrm{T} 3:>4.0\end{array}$} & T2 vs. T1: OR = $1.11(0.71-1.74)$ & \\
\hline & & & $\mathrm{T} 3$ vs. $\mathrm{T} 1: \mathrm{OR}=1.50(1.12-2.00)$ & \\
\hline & & & $p$ for trend $=0.007$ & \\
\hline \multirow{2}{*}{$\begin{array}{l}\text { Shive et al. } \\
2014 \text { [45] }\end{array}$} & \multirow{2}{*}{$\begin{array}{l}\text { High BP: systolic BP } \geq 140 \mathrm{mmHg} \\
\text { and diastolic BP } \geq 90 \mathrm{mmHg}\end{array}$} & \multirow[t]{2}{*}{ BPA continuous (log-transformed) } & Adjusted model: $\mathrm{OR}=1.14(1.00-1.30)$ & \multirow[t]{2}{*}{ Urinary creatinine, age at examination, sex, ethnicity, BMI } \\
\hline & & & $\begin{array}{l}\text { Weighted model (additionally adjusted for } \\
\text { subsample weighting): } \mathrm{OR}=1.12(0.93-1.35)\end{array}$ & \\
\hline
\end{tabular}

$B M I$ body mass index; $B P$ blood pressure; $B P A$ bisphenol $A ; C A D$ coronary artery disease; $C H D$ coronary heart disease; $C V D$ cardiovascular disease; $H D L$ high density lipoprotein; $L D L$ low density lipoprotein; $M I$ myocardial infraction; $O R$ odds ratio; $S D$ : standard deviation; $T G$ triglycerides; WC waist circumference 
corrected for renal function using the ratio of BPA-tocreatinine as the exposure variable [34, 54, 63, 64]. All but 4 adult studies adjusted for smoking [32, 51, 53, 58], and in the studies of children, smoking exposure was assessed by urine cotinine $[35,38,47,48,63,64]$ or questionnaire [64]. For the studies that investigated glucose, CVD or hypertension as the outcome, all except one [34] adjusted for WC [33, 37, 40, 46, 51], body mass index (BMI) [32, 33, 37-41, 43, 45, 46, 56, 59-61] or both height and weight [54].

All studies except 8 [32, 45, 50, 53, 54, 58, 61, 62] adjusted their statistical models for socioeconomic variables (e.g., education, income, occupation). All U.S. studies were adjusted for race/ethnicity.

Ten out of 33 studies (30 \%) adjusted their models for dietary intake such as total energy intake [33, 34, 47, 48, 62], sugar-sweetened soda consumption [38, 64], 'healthy' food consumption $[49,61,62]$ or unbalanced diet (e.g., fast food or sweet consumption) $[49,64]$. Casey \& Neidell adjusted for potential BPA sources including consumption of bottled water and canned tuna [37].

\section{Quality of individual studies}

Detailed results of the quality assessment of individual studies according to our scoring system are reported in the Additional file 1: Table S4. In summary, there were 4 (12\%) studies scored as 'high-quality', 27 (82 \%) scored as 'medium-quality' and 2 (6\%) scored as 'low-quality'. All 'high-quality' studies were longitudinal [60, 61, 63, 64]. Results reported in these 'high-quality' studies were mixed or significant. Significant and non-significant results were also reported in 'medium quality' and 'low quality' studies.

\section{Diabetes and hyperglycemia Diabetes}

Eight cross-sectional studies reported on the relation between uBPA and diabetes [32, 37, 39, 40, 42, 46, 51, 56], and seven supported a positive association (Table 2).

In NHANES, a positive association between uBPA concentrations and self-reported diabetes was reported in the NHANES 2003-04 cycle $(\mathrm{OR}=1.39,95 \% \mathrm{CI}$ : 1.21-1.60) [39], but results did not reach significance in the NHANES 2005-06 [40] nor 2007-08 [46] populations. Indeed, Casey \& Neidell found significant interactions between uBPA and NHANES cycle [37]. Using 3 different statistical models within NHANES cycles from 2003-04 to 2007-08, they reported a positive association in only the 2003-04 cycle.

In a pooled NHANES 2003-08 dataset, Shankar \& Teppala used a definition of diabetes based on fasting glucose level, HbA1c level, non-fasting glucose level and self-reported current use of diabetes medication, and found a significant positive linear trend across uBPA quartiles $(p=0.002)$ [42]. Although both statistically significant, the OR for quartile 4 (Q4) compared to quartile $1(\mathrm{Q} 1)$ was about two times higher in normal weight participants $(\mathrm{OR}=3.17)$ than in overweight/obese participants $(\mathrm{OR}=1.56)$.

No significant association was shown in a Korean crosssectional study [56]. In a Chinese cross-sectional study, the adjusted OR for type 2 diabetes was statistically significant in the second (OR $=1.30,95 \%$ CI: 1.03-1.64) and fourth $(\mathrm{OR}=1.37,95 \%$ CI: 1.08-1.74) uBPA quartiles, but not in the third uBPA quartile (OR=1.09, $95 \%$ CI: 0.86-1.39), compared to the first quartile [51]. Among 131 Iranian participants, Ahmadkhaniha et al. reported a significant adjusted OR of 57 for type 2 diabetes when comparing the participants with uBPA above the median to those with uBPA below the median [32].

One prospective study, among U.S. women from the NHS and the NHSII cohorts, has been published [61]. While a positive association between uBPA and incident diabetes was reported among middle-aged women from the NHSII study (OR $=2.08,95 \%$ CI: $1.17-3.69$, for Q4 vs. Q1), no significant results were found in older women from the NHS study $(\mathrm{OR}=0.81,95 \%$ CI: $0.48-1.38$, for Q4 vs. Q1).

\section{Prediabetes and hyperglycemia}

In one cross-sectional study, there was a positive association between uBPA tertiles and prediabetes, with associations stronger in women and obese subjects [41]. Two cross-sectional studies reported the relation between uBPA and elevated fasting blood glucose, one in children [38], one in adults [34]; neither reported a significant association.

\section{Meta-analysis}

For prevalent diabetes, the inclusion of the study by Ahmadkhaniha et al. [32] led to highly significant heterogeneity $\left(p<10^{-5}\right.$ and $\left.\mathrm{I}^{2}=94 \%\right)$; consequently we excluded this study from the meta-analysis. Finally, three cross-sectional studies, all examining uBPA levels in quartiles, were included in the meta-analysis $(n=9291)$ $[37,51,56]$. Pooled ORs (95 \% CI) were $1.33(1.10-$ $1.61), 1.18(0.97-1.44)$ and $1.47(1.21-1.80)$ in the second, third, and fourth uBPA quartiles compared to the first uBPA quartile. No significant heterogeneity was observed $\left(p=0.88,0.41\right.$ and 0.55 respectively, $\left.\mathrm{I}^{2}=0 \%\right)$. We were unable to conduct a meta-analysis for the other outcomes due to insufficient numbers of studies.

\section{Anthropometry and adiposity}

The relationships between uBPA and measures of anthropometry (e.g., weight, overweight, obesity) or adiposity (e.g., fat mass) were investigated in 16 studies, including 8 studies conducted in children (Table 3). 


\section{Overweight and obesity}

A positive cross-sectional association between uBPA and overweight was reported in 2 studies [49, 64], whereas 5 publications reported no significant associations with overweight $[36,38,47,52,55]$. In the only longitudinal study, uBPA concentrations at age 5 were not associated with overweight at age 9 [64]. All but one study found a significant positive cross-sectional association between higher uBPA concentrations and obesity [35, 38, 44, 47, 52, 55], with ORs ranging from 1.50 to 2.57 for $\mathrm{Q} 4$ vs. Q1.

\section{Waist circumference}

The four cross-sectional studies examining the relationship between uBPA and prevalent abdominal obesity in adults showed significant positive results [36, 44, 52, 57]. Eng et al. reported that in children, higher uBPA concentrations were significantly associated with elevated WC-to-height ratio (WHR), but not with elevated WC [38].

Three studies considered WC or WHR as a continuous variable. One showed a positive linear association between daily BPA excretion and WC [58] and another, in women, reported a significant positive correlation between uBPA concentrations and WC [53]. The only study in children found a statistically significant positive association between UBPA and WHR [48].

\section{Weight and BMI}

Three cross-sectional studies examined the association between UBPA and weight [58] or BMI [50,53] as continuous variables. Both Chinese studies by Wang et al. [50] and Zhao et al. [53] reported significant positive associations between uBPA and BMI. Galloway et al. [58] showed that daily BPA excretion was significantly and positively associated with BMI and weight in Italian adults.

Among the prospective studies, one reported that early childhood uBPA concentrations were associated with a modest and non-significant reduction in child BMI between 2 and 5 years of age [63], whereas the other found that higher uBPA concentrations were significantly associated with modestly faster weight gain in women [62].

\section{Adiposity}

In Chinese healthy premenopausal women, fat mass was significantly correlated with uBPA when adjusting for age [53]. In pooled NHANES 2003-10, abnormal body fat in children was not associated with uBPA concentrations in quartiles [38].

\section{Meta-analysis}

We included 5 cross-sectional studies for overweight $[36,38,49,52,64]$, including 2 in adults, 3 crosssectional studies for obesity [38, 44, 52], including 2 in adults, and 4 cross-sectional studies for elevated WC [38, 44, 52, 57], including 3 in adults.
Higher BPA exposure was significantly associated with obesity and elevated WC, and no significant heterogeneity between studies was found (Table 5). There was evidence for overweight only in adults.

\section{Cardiovascular disease and hypertension Cardiovascular disease}

Four of the five cross-sectional studies reported a positive linear association between UBPA and CVD (Table 4). An increased risk of self-reported CVD (myocardial infarction, angina, or coronary heart disease [CHD]: alone or combined) was associated with increased concentration of uBPA (OR $=1.39,95 \% \mathrm{CI}: 1.18-1.63)$, but no increased risk of stroke (OR $=0.97,95 \% \mathrm{CI}$ : 0.74-1.27) in NHANES 2003-04 [39]. Melzer et al. then reported similar associations when including NHANES 2005-06 data [40]. LaKind et al. examined NHANES data from $2003-04$ to $2009-10$ in adults aged $\geq 20$ years, and found no significant associations between self-reported CHD, heart attack and uBPA as a continuous variable in either separate or pooled populations [33]. Casey \& Neidell reported significant positive associations between uBPA and CHD in NHANES 2003-04 but results were not consistent in the subsequent 2005/06 and 2007/08 cycles [37]. Melzer et al. studied coronary artery disease (CAD) severity assessed by angiography [59]. Compared to participants without CAD $(n=120)$, uBPA concentrations per standard deviation (SD) increase - were significantly

Table 5 Pooled OR estimates for diabetes, overweight, obesity, elevated waist circumference and hypertension comparing extreme categories of urinary BPA levels (the highest vs. the lowest): random effect models

\begin{tabular}{|c|c|c|c|c|}
\hline \multirow[t]{2}{*}{ Outcome } & \multirow{2}{*}{$\begin{array}{l}\text { Number } \\
\text { of } \\
\text { studies }\end{array}$} & \multirow{2}{*}{$\begin{array}{l}\text { Pooled OR } \\
(95 \% \mathrm{Cl})\end{array}$} & \multicolumn{2}{|c|}{ Heterogeneity } \\
\hline & & & $p$-value & $\mathrm{I}^{2}(\%)$ \\
\hline Prevalent diabetes & 3 & $1.47(1.21-1.80)$ & 0.55 & 0 \\
\hline \multicolumn{5}{|l|}{ Prevalent overweight } \\
\hline Total & 5 & $1.21(0.98-1.50)$ & 0.09 & 45 \\
\hline Children only & 3 & $1.24(0.88-1.75)$ & 0.03 & 62 \\
\hline Adults only & 2 & $1.25(1.01-1.56)$ & 0.84 & 0 \\
\hline \multicolumn{5}{|l|}{ Prevalent obesity } \\
\hline Total & 3 & $1.67(1.41-1.98)$ & 0.44 & 0 \\
\hline Adults only & 2 & $1.60(1.32-1.93)$ & 0.54 & 0 \\
\hline \multicolumn{5}{|l|}{ Prevalent elevated WC } \\
\hline Total & 4 & $1.48(1.25-1.76)$ & 0.28 & 21 \\
\hline Adults only & 3 & $1.52(1.21-1.90)$ & 0.15 & 47 \\
\hline Prevalent hypertension & 2 & $1.41(1.12-1.79)$ & 0.50 & 0 \\
\hline
\end{tabular}

BPA bisphenol A; $C$, confidence interval; $O R$ odds ratio; $W C$ waist circumference

${ }^{a}$ Using ORs comparing extreme categories of urinary BPA levels (the highest vs. the lowest) summarized in Tables 2, 3 and 4 
associated with severe CAD (OR $=1.43,95 \%$ CI: 1.03-1.98; $n=385$ ), and near significantly associated with intermediate CAD (OR $=1.69,95$ \% CI: $0.98-2.94 ; n=86)$.

The only prospective study, a nested case-control study within the EPIC-Norfolk cohort, reported a positive association between uBPA concentrations and incidence of CAD up to 10 years after BPA measurement, with a significant increase in risk of CAD per SD increase in uBPA: $\mathrm{OR}=1.11,95 \% \mathrm{CI}=1.00-1.23$ [60].

\section{Hypertension}

Three cross-sectional studies examined the association between uBPA and hypertension (Table 4). Both Shankar \& Teppala (using NHANES 2003-04 data) [43] and Shiue et al. (NHANES 2009-10) [45] showed a positive association between $\mathrm{UBPA}$ and hypertension. Bae et al. reported a positive but non-significant association between uBPA and hypertension ( $\mathrm{OR}=1.27$, 95 \% CI: $0.85-1.88$ for Q4 vs. Q1) in an elderly Korean population, and the association reached significance when the analyses were restricted to participants without a previous history of hypertension $(\mathrm{OR}=2.35,95 \%$ CI: 1.33-4.17) [54].

\section{Meta-analysis}

Meta-analysis for the CVD and UBPA data was not possible owing to different study designs and use of overlapping NHANES data. For hypertension, we included two cross-sectional studies and the pooled OR was statistically significant (Table 5).

\section{Discussion}

\section{Summary of evidence}

Our analysis presents summarized evidence of BPA exposure and its associations with chronic cardiometabolic disorders including glucose abnormalities, measures of overweight/obesity, CVD and hypertension in humans.

Of the 33 epidemiological studies included, results were generally consistent across cross-sectional studies, with positive associations between uBPA concentrations and diabetes, general obesity, abdominal obesity, CVD and hypertension suggested in $7 / 8,6 / 7,5 / 5,4 / 5$ and $2 / 3$ publications respectively. We were able to conduct outcome-specific meta-analysis including 12 independent studies. While a risk of bias cannot be excluded, the results indicate positive cross-sectional associations between uBPA concentrations and diabetes, general obesity, abdominal obesity and hypertension. Results were significant for overweight in adults but not in children. We were unable to examine uBPA and CVD in metaanalysis due to overlapping data and/or heterogeneity among studies (in terms of study design and definition of outcome).

Among the 5 prospective studies included in this review, 3 showed that uBPA levels were positively associated with incident type 2 diabetes and CAD, and weight gain. While these first prospective results seem to corroborate findings from cross-sectional studies, more prospective data are needed to make the evidence more robust.

\section{Strengths and limitations of the current review}

In recent years, there has been a rapid increase in publications examining the possible relationship between BPA exposure and cardiometabolic health. A systematic review of the epidemiological evidence linking BPA with indicators of obesity, glucose metabolism and CVD, was published by LaKind et al. in 2014 [33]. Further to their results, we have been able to include 11 further publications into this review. We have also calculated pooled ORs for diabetes, overweight, general/abdominal obesity, and hypertension. In their systematic review, LaKind et al. concluded that meta-analysis was not feasible due to the heterogeneity across studies. Nonetheless we were able to identify small but significant groups of crosssectional studies similar enough to be included in a meta-analysis. However it should be noted that studies included in the meta-analyses can differ with regard to some characteristics, such as outcome ascertainment (especially for diabetes), adjustment variables, and exposure contrast.

As with many reviews of observational data, the risk of publication bias cannot be completely ruled out and it can be hypothesized that studies with significant and positive results are more widely disseminated than those with non-significant and/or negative results. We considered producing funnel plots to explore the presence of publication bias across studies included in the metaanalyses. However, as a rule of thumb, tests for funnel plot asymmetry should not be used when there are fewer than 10 studies in the meta-analysis because test power is usually too low to distinguish chance from real asymmetry [65]. We restricted this review to studies published in English, due to the generalization of the English language in scientific publications. To ascertain if we may have omitted studies not published in English, we ran the same search in PubMed, but limiting the results to non-English languages. We found 12 new articles (5 in Chinese, 3 in Japanese, 2 in Italian, 1 in Spanish, and 1 in Czech), but based on abstract, none of them met the eligibility criteria other than language.

\section{Strengths and limitations of studies included in the review}

Most studies were not designed with environmental exposures in mind. Even though there is evidence for a cross-sectional relation between UBPA and cardiometabolic disorders, results were different across studies, even in different cycles from the same study (NHANES). 
Depending on the studies, possible explanations could be incomplete adjustment for confounders, measurement error, or differences in study design.

When studying the relation between BPA exposure and cardiometabolic disorders, the statistical models should be properly adjusted for confounding variables. Dietary intake, especially high levels of processed food, has to be considered as a major potential confounding factor for the relationship between uBPA and metabolic disease. It has been suggested that people with diabetes or obesity may consume more food, either in absolute volume or from BPA-containing packaging (plastic containers, polycarbonate drink bottles, tin cans coated with epoxy resin, etc.), which may result in higher uBPA concentrations than non-diabetic or healthy weight individuals. Therefore we can suggest that positive findings observed in cross-sectional studies may be due to dietary sources of BPA exposure that are also important predictors of adiposity. Less than one-third of studies included in this review controlled for diet, mostly via total daily energy intake, and only 1 for possible dietary sources of BPA exposure (canned tuna fish and bottled water consumption). Thus, there is a strong need for observational studies controlling for dietary characteristics including potential sources of BPA exposure. Moreover, food and drink in BPA-containing packaging are not necessarily similar in terms of nutritional value (e.g., canned vegetables compared to processed meat, or bottled water compared to sugar-sweetened beverages). Consequently, there is a requirement for data on both dietary patterns (e.g., overall energy intake) along with sources of dietary exposure to BPA, as this helps disentangle real associations from confounding.

Additionally, given the potential correlation of environmental toxicants with each other, exposure to other EDCs may be another confounding factor. Two studies controlled for exposure to other environmental phenols [47] or to phthalates [37]. In both studies, when added to final multivariable models, the authors reported that the other contaminants did not substantially change the associations of uBPA concentrations with the studied outcomes.

Other methodological limitations of studies relate to BPA exposure assessment. BPA concentrations have been mostly determined in a single urine measurement. There is accumulating evidence that a single measurement may not reflect chronic exposure to BPA: regardless of the timing of urine sampling (spot, first morning void, 24 h collection), BPA concentrations were shown, in individual adults, to considerably vary throughout the day and the week [66]. Thus, using only a single uBPA measurement may lead to non-differential exposure misclassification of the participants and bias the estimates. The study by Snijder et al., in pregnant women, showed that using only one uBPA measurement resulted in underestimation of the association between maternal uBPA and fetal growth, which was stronger and significant when several measurements were taken into account [67]. Consequently, it would be better to increase the number of measurements per participant. However, in the context of large-scale epidemiological studies, the substantial cost of the BPA assay is clearly a limitation. Interestingly, most studies report only a single urinary measurement yet the majority show positive associations between uBPA levels and the outcomes of interest.

Another controversy that exists in the BPA literature relates to the lack of knowledge on 'deep' compartments, which may serve as potential sources of BPA exposure. Before being metabolized by the liver, BPA is a lipophilic compound. Although it was thought to be rapidly metabolized and excreted, recent data showed a half-life longer than expected, suggesting a potential accumulation in body tissues such as adipose tissue [68]. In agreement with this hypothesis, Fernandez et al. showed, among 20 Spanish women, that unconjugated BPA was detected in adipose tissue in $55 \%$ of the samples [69].

The other key issue in the human data is the lack of prospective data, reflecting the inherent difficulties in performing longitudinal studies, which are costly and time-consuming. Five studies used a prospective design and the main outcomes differ. Although literature in this area is rapidly increasing, the majority of studies published have, and continue to be, of a cross-sectional nature. Indeed, among the 11 studies published between 1 June 2013 and 1 August 2014, only 3 have a prospective design. Nearly half of the publications included in this review relied on NHANES cross-sectional data, including a substantial number of publications using the same study population. Consequently, it is imperative that more prospective epidemiological data is presented, especially with outcomes related to glucose tolerance and anthropometry, which appear to show the most consistent associations. Certainly, given chronic diseases such as diabetes develop over a timespan of many years, timing of BPA measurement would need to occur within an etiologically relevant window before diagnosis of the disease.

Differences in analyses and results contribute to confusion about the association between BPA exposure and cardiometabolic disorders. As Casey \& Neidell highlighted, using continuous uBPA, continuous log-transformed uBPA or uBPA in quartiles as exposure variable can lead to lack of consistency in results in a same data set [37]. It therefore seems difficult to draw definitive conclusions from the available data, and publications may be vulnerable to biases given that the same data set can produce significant or non-significant associations according to the different statistical models utilized. 
At present, the "gold standard" method for testing BPA is solid-phase extraction coupled with HPLC-MS/ MS with peak focusing [13]. Overall, chromatographybased methods are expensive and, thus, not particularly suitable for the large numbers of samples which are required to ensure that analyses are adequately powered to detect any association. Less expensive methods have been proposed, focusing on BPA metabolites (e.g., BPAglucuronide) [70, 71]. It has been suggested that part of the BPA measured in human samples can be due to contamination during sample collection or laboratory measurements [72, 73], and one of the great advantages of studying BPA metabolites is to avoid these contamination issues. The lower cost of these methods would also allow measuring BPA in larger populations. However, at present, the use of urinary levels of BPA metabolites as surrogates for BPA exposure remains to be validated.

\section{Future research and implications}

Experimental studies have suggested that BPA exposure is associated with abnormal glucose metabolism and insulin resistance. However, data from observational studies of humans supporting an association between BPA and obesity, diabetes and CVD are still too limited in cross-sectional studies to make definitive statements of harm. It is imperative that more prospective studies, with careful measurements of dietary intake, socioeconomic status and urine dilution, are conducted to understand the potential impact BPA exposure in humans will have on the development of chronic disease. Concerning the health outcomes of interest, if associations with overweight/obesity defined according to BMI have been much studied, there are a limited number of studies using more accurate measures of adiposity (such as percent body fat or fat mass). In some studies, analyses were stratified by gender, age, BMI or pre-existing disease. Future studies should continue to explore the existence of populations potentially at greatest risk for BPA-related health effects, in order to confirm or refute these first results. Irrespective of outcome, if confirmed, reasons for differences in these associations will need further exploration. Certainly, no epidemiological study has aimed to identify critical windows of exposure and the risk of incident cardiometabolic disorders. For chronic diseases which have a long latent period, different lag times should be tested. This should feature in future longitudinal studies, especially in paediatric cohorts.

Even in the absence of certainty, there are growing community concerns about the possible deleterious impact of BPA on human health. In agreement with the precautionary principle, regulations have been adopted by a number of countries, prohibiting BPA in baby feeding bottles, in food containers for young children, or in all food and drink packaging, with the aim of reducing human exposure to BPA [74]. If the hypothesised associations are confirmed, this will not only provide evidence to further limit or ban the use of BPA in the food industry, but also advance the impetus to research other health outcomes, and to further explore the impact of a broad range of potential environmental toxins on chronic disease. Lastly, given the recent efforts to reduce BPA exposure, finding safe alternatives to BPA is a very important issue. While BPA can be replaced by structurally similar molecules, such as other bisphenols (e.g., bisphenol $\mathrm{S}$, bisphenol F), initial studies suggest similar endocrine disrupting effects [75], and epidemiological studies should now also consider exposure to BPA-replacement chemicals.

\section{Conclusion}

In summary, we have shown that uBPA at levels found in the general population is associated with increased prevalence of diabetes, general and abdominal obesity, and hypertension. Additional prospective data are needed to ascertain the nature of the relationship between BPA exposure and cardiometabolic disorders. Prospective cohort studies, with carefully collected data on dietary sources of BPA exposure and other potential confounders, as well as repeated uBPA measurements, are indicated and will likely prove useful in filling this gap in the literature and clarifying/explicating these complex relationships.

\section{Additional file}

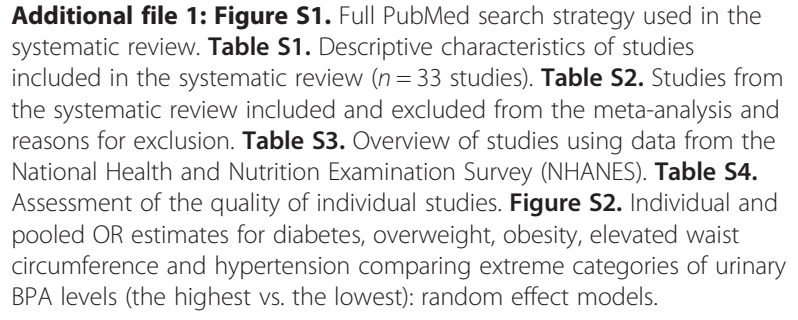

Additional file 1: Figure S1. Full PubMed search strategy used in the systematic review. Table S1. Descriptive characteristics of studies included in the systematic review ( $n=33$ studies). Table S2. Studies from the systematic review included and excluded from the meta-analysis and reasons for exclusion. Table S3. Overview of studies using data from the National Health and Nutrition Examination Survey (NHANES). Table S4. Assessment of the quality of individual studies. Figure S2. Individual and pooled OR estimates for diabetes, overweight, obesity, elevated waist circumference and hypertension comparing extreme categories of urinary BPA levels (the highest vs. the lowest): random effect models.

\section{Abbreviations}

ADA: American Diabetes Association; ALT: Alanine aminotransferase BMI: Body mass index; BP: Blood pressure; BPA: Bisphenol A; CAD: Coronary artery disease; CHAMACOS: Center for the health assessment of mothers and children of Salinas; CHD: Coronary heart disease; Cl: Confidence interval; CVD: Cardiovascular disease; EDCs: Endocrine-disrupting chemicals; eGFR: Estimated glomerular filtration rate; EPIC: European prospective investigation into cancer; ER: Estrogen-related receptor; GGT: Gamma glutamyltransferase; HOMA: Homeostasis model of assessment; HOME: Health outcomes and measures of the environment; HDL: High density lipoprotein; HPLC-MS/MS: High performance liquid chromatographytandem mass spectrometry; HR: Hazard ratio; hsCRP: High-sensitivity Creactive protein; IQR: Interquartile range; LDL: Low density lipoprotein; LOD: Limit of detection; LOQ: Limit of quantification; NHANES: National health and nutrition examination survey; NHS: Nurses' health study; OR: Odds ratio; PPAR: Peroxisome proliferation agonist receptor; PRISMA: Preferred reporting items for systematic reviews and meta-analyses; Q1: First quartile; Q4: Fourth quartile; TG: Triglycerides; SD: Standard deviation; SE: Standard error; uBPA: Urinary bisphenol A; WC: Waist circumference; WHR: Waist circumference-to-height ratio. 


\section{Competing interests}

The authors declare that they have no competing interests.

\section{Authors' contributions}

$F R, J L, V L$ and DJM participated in literature searching, data extraction and data analysis and drafted the manuscript. JB, TG, TW, JES critically revised the manuscript for important intellectual content. All authors approved the final version.

\section{Acknowledgments}

This study was funded by the National Health and Medical Research Council, Australia (NHMRC grant APP1022923). Support for FR was provided by a postdoctoral grant from CORDDIM (field of major interest of the Île-deFrance Regional Council "Cardiovascular/Obesity/Kidney/Diabetes"), the French Endocrine Disruptor Research Programme (PNRPE grant) and the Scientific Mobility Program of the Embassy of France in Australia (2014). JGL is supported by National Heart Foundation of Australia/NHMRC postgraduate scholarship [586739] and the Cardiac Society of Australia and New Zealand. This work was supported in part by the Victorian Government's OIS Program.

\section{Author details}

${ }^{1}$ Inserm, U1153, Epidemiology and Biostatistics Sorbonne Paris Cité Research Centre (CRESS), Early Origin of the Child's Health and Development (ORCHAD) Team, Villejuif, France. ²Univ Paris Descartes, UMR1153, Paris, France. ${ }^{3}$ Department of Clinical Diabetes and Epidemiology, Baker IDI Heart and Diabetes Institute, Level 4, 99 Commercial Road, Melbourne, VIC 3000, Australia. ${ }^{4}$ Faculty of Pharmacy, Univ Paris-Sud, Châtenay-Malabry, France. ${ }^{5}$ Department of Biosciences, University of Exeter, College of Life and Environmental Sciences, Exeter, UK. ${ }^{6}$ Shanghai Clinical Center for Endocrine and Metabolic Diseases, Rui Jin Hospital, Shanghai Jiao Tong University School of Medicine, Shanghai, China.

\section{Received: 5 January 2015 Accepted: 25 May 2015}

\section{Published online: 31 May 2015}

\section{References}

1. Magliano DJ, Lyons JG. Bisphenol A and diabetes, insulin resistance, cardiovascular disease and obesity: controversy in a (plastic) cup? J Clin Endocrinol Metab. 2013;98:502-4.

2. Longnecker MP, Daniels JL. Environmental contaminants as etiologic factors for diabetes. Environ Health Perspect. 2001;109 Suppl 6:871-6.

3. Vom Saal FS, Akingbemi BT, Belcher SM, Birnbaum LS, Crain DA, Eriksen M, et al. Chapel Hill bisphenol A expert panel consensus statement: integration of mechanisms, effects in animals and potential to impact human health at current levels of exposure. Reprod Toxicol. 2007;24:131-8.

4. Elobeid MA, Allison DB. Putative environmental-endocrine disruptors and obesity: a review. Curr Opin Endocrinol Diabetes Obes. 2008;15:403-8.

5. Thayer KA, Heindel JJ, Bucher JR, Gallo MA. Role of environmental chemicals in diabetes and obesity: a National Toxicology Program workshop review. Environ Health Perspect. 2012;120:779-89.

6. FitzGerald RE, Wilks MF. Bisphenol A-why an adverse outcome pathway framework needs to be applied. Toxicol Lett. 2014;230:368-74.

7. Huang YQ, Wong CKC, Zheng JS, Bouwman H, Barra R, Wahlström B, et al. Bisphenol A (BPA) in China: a review of sources, environmental levels, and potential human health impacts. Environ Int. 2012;42:91-9.

8. Vandenberg LN, Hauser R, Marcus M, Olea N, Welshons WV. Human exposure to bisphenol A (BPA). Reprod Toxicol. 2007;24:139-77.

9. Geens T, Aerts D, Berthot C, Bourguignon JP, Goeyens L, Lecomte P, et al. A review of dietary and non-dietary exposure to bisphenol-A. Food Chem Toxicol. 2012;50:3725-40.

10. Porras SP, Heinälä M, Santonen T. Bisphenol A exposure via thermal paper receipts. Toxicol Lett. 2014;230:413-20.

11. Hormann AM, Vom Saal FS, Nagel SC, Stahlhut RW, Moyer CL, Ellersieck MR, et al. Holding thermal receipt paper and eating food after using hand sanitizer results in high serum bioactive and urine total levels of bisphenol A (BPA). PLoS One. 2014;9:e110509.

12. Rudel RA, Gray JM, Engel CL, Rawsthorne TW, Dodson RE, Ackerman JM, et al. Food packaging and bisphenol A and bis(2-ethyhexyl) phthalate exposure: findings from a dietary intervention. Environ Health Perspect. 2011;119:914-20.
13. Vandenberg LN, Chahoud I, Heindel JJ, Padmanabhan V, Paumgartten FJR, Schoenfelder G. Urinary, circulating, and tissue biomonitoring studies indicate widespread exposure to bisphenol A. Environ Health Perspect. 2010;118:1055-70

14. Völkel W, Colnot T, Csanády GA, Filser JG, Dekant W. Metabolism and kinetics of bisphenol $A$ in humans at low doses following oral administration. Chem Res Toxicol. 2002;15:1281-7.

15. Geens T, Neels H, Covaci A. Distribution of bisphenol-A, triclosan and nnonylphenol in human adipose tissue, liver and brain. Chemosphere. 2012;87:796-802.

16. Fenichel P, Chevalier N, Brucker-Davis F. Bisphenol A: an endocrine and metabolic disruptor. Ann Endocrinol (Paris). 2013;74:211-20.

17. Delfosse V, Grimaldi M, Cavaillès V, Balaguer P, Bourguet W. Structural and functional profiling of environmental ligands for estrogen receptors. Environ Health Perspect. 2014;122:1306-13.

18. Alonso-Magdalena P, Morimoto S, Ripoll C, Fuentes E, Nadal A. The estrogenic effect of bisphenol A disrupts pancreatic $\beta$-cell function in vivo and induces insulin resistance. Environ Health Perspect. 2005;114:106-12.

19. Alonso-Magdalena P, Quesada I, Nadal A. Endocrine disruptors in the etiology of type 2 diabetes mellitus. Nat Rev Endocrinol. 2011;7:346-53.

20. Alonso-Magdalena P, Ropero AB, Soriano S, Quesada I, Nadal A. Bisphenol-A: a new diabetogenic factor? Hormones. 2010;9:118-26.

21. Alonso-Magdalena P, Ropero AB, Carrera MP, Cederroth CR, Baquié M, Gauthier BR, et al. Pancreatic insulin content regulation by the estrogen receptor ER alpha. PLoS One. 2008;3:e2069.

22. Soriano S, Alonso-Magdalena P, García-Arévalo M, Novials A, Muhammed SJ, Salehi A, et al. Rapid insulinotropic action of low doses of bisphenol-A on mouse and human islets of Langerhans: role of estrogen receptor $\beta$. PLoS One. 2012;7:e31109.

23. Hugo ER, Brandebourg TD, Woo JG, Loftus J, Alexander JW, Ben-Jonathan N. Bisphenol $A$ at environmentally relevant doses inhibits adiponectin release from human adipose tissue explants and adipocytes. Environ Health Perspect. 2008;116:1642-7.

24. Andersson $\mathrm{H}$, Brittebo E. Proangiogenic effects of environmentally relevant levels of bisphenol A in human primary endothelial cells. Arch Toxicol. 2012;86:465-74.

25. Marmugi A, Lasserre F, Beuzelin D, Ducheix S, Huc L, Polizzi A, et al. Adverse effects of long-term exposure to bisphenol A during adulthood leading to hyperglycemia and hypercholesterolemia in mice. Toxicology. 2014;325:133-43.

26. Rochester JR. Bisphenol A, and human health: a review of the literature. Reprod Toxicol. 2013;42:132-55.

27. Rezg R, El-Fazaa S, Gharbi N, Mornagui B. Bisphenol A and human chronic diseases: current evidences, possible mechanisms, and future perspectives. Environ Int. 2013;64C:83-90.

28. Lakind JS, Goodman M, Mattison DR. Bisphenol A and indicators of obesity, glucose metabolism/type 2 diabetes and cardiovascular disease: a systematic review of epidemiologic research. Crit Rev Toxicol. 2014;44:121-50.

29. Shojania KG, Sampson M, Ansari MT, Ji J, Garritty C, Rader T, et al. Updating systematic reviews. In: AHRQ Technical Reviews, vol. 16. Rockville, MD: Agency for Healthcare Research and Quality (US); 2007.

30. Moher D, Liberati A, Tetzlaff J, Altman DG, PRISMA Group. Preferred reporting items for systematic reviews and meta-analyses: the PRISMA statement. J Clin Epidemiol. 2009;62:1006-12.

31. Rooney AA, Boyles AL, Wolfe MS, Bucher JR, Thayer KA. Systematic review and evidence integration for literature-based environmental health science assessments. Environ Health Perspect. 2014;122:711-8.

32. Ahmadkhaniha R, Mansouri M, Yunesian M, Omidfar K, Jeddi MZ, Larijani B, et al. Association of urinary bisphenol a concentration with type-2 diabetes mellitus. J Environ Health Sci Eng. 2014;12:64.

33. LaKind JS, Goodman M, Naiman DQ. Use of NHANES data to link chemical exposures to chronic diseases: a cautionary tale. PLoS One. 2012;7:e51086.

34. Beydoun HA, Khanal S, Zonderman AB, Beydoun MA. Sex differences in the association of urinary bisphenol-A concentration with selected indices of glucose homeostasis among U.S. adults. Ann Epidemiol. 2014;24:90-7.

35. Bhandari R, Xiao J, Shankar A. Urinary bisphenol A and obesity in U.S. children. Am J Epidemiol. 2013;177:1263-70.

36. Carwile JL, Michels KB. Urinary bisphenol A and obesity: NHANES 2003-2006. Environ Res. 2011;111:825-30.

37. Casey MF, Neidell M. Disconcordance in statistical models of bisphenol A and chronic disease outcomes in NHANES 2003-08. PLOS One. 2013;8:e79944 
38. Eng DS, Lee JM, Gebremariam A, Meeker JD, Peterson K, Padmanabhan V. Bisphenol A and chronic disease risk factors in US children. Pediatrics. 2013;132:e637-45.

39. Lang IA, Galloway TS, Scarlett A, Henley WE, Depledge M, Wallace RB, et al. Association of urinary bisphenol A concentration with medical disorders and laboratory abnormalities in adults. JAMA. 2008;300:1303-10.

40. Melzer D, Rice NE, Lewis C, Henley WE, Galloway TS. Association of urinary bisphenol A concentration with heart disease: evidence from NHANES 2003/06. PLoS One. 2010;5:e8673.

41. Sabanayagam C, Teppala S, Shankar A. Relationship between urinary bisphenol A levels and prediabetes among subjects free of diabetes. Acta Diabetol. 2013;50:625-31.

42. Shankar A, Teppala S. Relationship between urinary bisphenol A levels and diabetes mellitus. J Clin Endocrinol Metab. 2011;96:3822-6.

43. Shankar A, Teppala S. Urinary bisphenol A and hypertension in a multiethnic sample of US adults. J Environ Public Health. 2012;2012:481641.

44. Shankar A, Teppala S, Sabanayagam C. Urinary bisphenol A levels and measures of obesity: results from the National Health and Nutrition Examination Survey 2003-2008. ISRN Endocrinol. 2012;2012:965243.

45. Shiue I. Higher urinary heavy metal, arsenic, and phthalate concentrations in people with high blood pressure: US NHANES, 2009-2010. Blood Press. 2014;23:363-9.

46. Silver MK, O'Neill MS, Sowers MR, Park SK. Urinary bisphenol A and type-2 diabetes in U.S. adults: data from NHANES 2003-2008. PLoS One. 2011;6:e26868.

47. Trasande L, Attina TM, Blustein J. Association between urinary bisphenol A concentration and obesity prevalence in children and adolescents. JAMA. 2012;308:1113-21.

48. Wells EM, Jackson LW, Koontz MB. Association between bisphenol A and waist-to-height ratio among children: National Health and Nutrition Examination Survey, 2003-2010. Ann Epidemiol. 2014;24:165-7.

49. Li DK, Miao M, Zhou Z, Wu C, Shi H, Liu X, et al. Urine bisphenol-A level in relation to obesity and overweight in school-age children. PLoS One. 2013;8:e65399.

50. Wang H, Zhou Y, Tang C, Wu J, Chen Y, Jiang Q. Association between bisphenol $\mathrm{A}$ exposure and body mass index in Chinese school children: a cross-sectional study. Environ Health. 2012;11:79.

51. Ning $G, B i$ Y, Wang $T, X u M, X u Y$, Huang $Y$, et al. Relationship of urinary bisphenol A concentration to risk for prevalent type 2 diabetes in Chinese adults: a cross-sectional analysis. Ann Intern Med. 2011;155:368-74.

52. Wang $T$, Li M, Chen B, Xu M, Xu Y, Huang Y, et al. Urinary bisphenol A (BPA) concentration associates with obesity and insulin resistance. J Clin Endocrinol Metab. 2012;97:E223-7.

53. Zhao H, Bi Y, Ma L, Zhao L, Wang $T$, Zhang $L$, et al. The effects of bisphenol A (BPA) exposure on fat mass and serum leptin concentrations have no impact on bone mineral densities in non-obese premenopausal women. Clin Biochem. 2012;45:1602-6.

54. Bae S, Kim JH, Lim YH, Park HY, Hong YC. Associations of bisphenol A exposure with heart rate variability and blood pressure. Hypertension. 2012;60:786-93.

55. Kim K, Park H, Yang W, Lee JH. Urinary concentrations of bisphenol A and triclosan and associations with demographic factors in the Korean population. Environ Res. 2011;111:1280-5.

56. Kim K, Park H. Association between urinary concentrations of bisphenol A and type 2 diabetes in Korean adults: a population-based cross-sectional study. Int J Hyg Environ Health. 2013;216:467-71.

57. Ko A, Hwang MS, Park JH, Kang HS, Lee HS, Hong JH. Association between urinary bisphenol $\mathrm{A}$ and waist circumference in Korean adults. Toxicol Res. 2014;30:39-44.

58. Galloway T, Cipelli R, Guralnik J, Ferrucci L, Bandinelli S, Corsi AM, et al. Daily bisphenol A excretion and associations with sex hormone concentrations: results from the InCHIANTI adult population study. Environ Health Perspect. 2010;118:1603-8.

59. Melzer D, Gates P, Osborne NJ, Henley WE, Cipelli R, Young A, et al. Urinary bisphenol A concentration and angiography-defined coronary artery stenosis. PLoS One. 2012;7:e43378.

60. Melzer D, Osborne NJ, Henley WE, Cipelli R, Young A, Money C, et al. Urinary bisphenol A concentration and risk of future coronary artery disease in apparently healthy men and women. Circulation. 2012;125:1482-90.

61. Sun Q, Cornelis MC, Townsend MK, Tobias DK, Eliassen AH, Franke AA, et al. Association of urinary concentrations of bisphenol $A$ and phthalate metabolites with risk of type 2 diabetes: a prospective investigation in the Nurses' Health Study (NHS) and NHSII cohorts. Environ Health Perspect. 2014; 122:616-23.

62. Song Y, Hauser R, Hu FB, Franke A, Liu S, Sun Q. Urinary concentrations of bisphenol $A$ and phthalate metabolites and weight change: a prospective investigation in US women. Int J Obes. 2014;38:1532-7.

63. Braun JM, Lanphear BP, Calafat AM, Deria S, Khoury J, Howe CJ, et al. Early-life bisphenol A exposure and child body mass index: a prospective cohort study. Environ Health Perspect. 2014;122:1239-45.

64. Harley KG, Aguilar Schall R, Chevrier J, Tyler K, Aguirre H, Bradman A, et al. Prenatal and postnatal bisphenol $\mathrm{A}$ exposure and body mass index in childhood in the CHAMACOS cohort. Environ Health Perspect. 2013;121:514-20.

65. Sterne JAC, Sutton AJ, loannidis JPA, Terrin N, Jones DR, Lau J, et al. Recommendations for examining and interpreting funnel plot asymmetry in meta-analyses of randomised controlled trials. BMJ. 2011;343:d4002.

66. Ye $X$, Wong LY, Bishop AM, Calafat AM. Variability of urinary concentrations of bisphenol $A$ in spot samples, first morning voids, and 24-hour collections. Environ Health Perspect. 2011;119:983-8.

67. Snijder CA, Heederik D, Pierik FH, Hofman A, Jaddoe W, Koch HM, et al. Fetal growth and prenatal exposure to bisphenol A: the generation $\mathrm{R}$ study. Environ Health Perspect. 2013;121:393-8.

68. Stahlhut RW, Welshons WV, Swan SH. Bisphenol A data in NHANES suggest longer than expected half-life, substantial nonfood exposure, or both. Environ Health Perspect. 2009;117:784-9.

69. Fernandez MF, Arrebola JP, Taoufiki J, Navalón A, Ballesteros O, Pulgar R, et al. Bisphenol-A and chlorinated derivatives in adipose tissue of women. Reprod Toxicol. 2007;24:259-64.

70. Lacroix MZ, Puel S, Collet SH, Corbel T, Picard-Hagen N, Toutain PL, et al. Simultaneous quantification of bisphenol $\mathrm{A}$ and its glucuronide metabolite (BPA-G) in plasma and urine: applicability to toxicokinetic investigations. Talanta. 2011;85:2053-9.

71. Harthé C, Rinaldi S, Achaintre D, de Ravel MR, Mappus E, Pugeat M, et al. Bisphenol A-glucuronide measurement in urine samples. Talanta. 2012;100:410-3.

72. Markham DA, Waechter Jr JM, Wimber M, Rao N, Connolly P, Chuang JC, et al. Development of a method for the determination of bisphenol $A$ at trace concentrations in human blood and urine and elucidation of factors influencing method accuracy and sensitivity. J Anal Toxicol. 2010;34:293-303.

73. Vandentorren S, Zeman F, Morin L, Sarter H, Bidondo ML, Oleko A, et al. Bisphenol-A and phthalates contamination of urine samples by catheters in the Elfe pilot study: implications for large-scale biomonitoring studies. Environ Res. 2011;111:761-4.

74. Udovyk O. Models of science-policy interaction: exploring approaches to bisphenol A management in the EU. Sci Total Environ. 2014;485-486:23-30.

75. Rochester JR, Bolden AL. Bisphenol S and F: a systematic review and comparison of the hormonal activity of bisphenol A substitutes. Environ Health Perspect. 2015; in press.

\section{Submit your next manuscript to BioMed Central and take full advantage of:}

- Convenient online submission

- Thorough peer review

- No space constraints or color figure charges

- Immediate publication on acceptance

- Inclusion in PubMed, CAS, Scopus and Google Scholar

- Research which is freely available for redistribution 